\title{
Gravitational Lensing by Black Holes
}

\author{
Valerio Bozza
}

Received: date / Accepted: date

\section{Introduction}

Whenever bending of light by gravitational fields is detected in astronomical observations it is generally very weak. Even in the spectacular formation of giant arcs, typical of the so-called strong lensing, photons are deflected by no more than a few arcseconds. This leads to the conclusion that the first order Einstein formula for the deflection angle is sufficient to explain all observed phenomenology. On the other hand, the existence of very compact objects, such as neutron stars or black holes, is now well-established through many independent astrophysical observations. In the neighbourhood of such objects, electromagnetic radiation is generated and travels through very strong gravitational fields. In such extreme cases, the calculation of the deflection of photons needs to be pushed beyond the first order approximation on which the Einstein formula relies. It must be said that no direct observation of gravitational lensing by black holes or other compact objects has ever been performed up to date. Yet, some peculiarities of the emission spectra of black holes, such as the shape of the $K_{\alpha}$ line of the iron, have been interpreted as the final effect of the strong deflection of photons emitted by the accretion disk.

The aim of this article is to review the theoretical aspects of gravitational lensing by black holes, and discuss the perspectives for realistic observations. We will first treat lensing by spherically symmetric black holes, in which the formation of infinite sequences of higher order images emerges in the clearest way. We will then consider the effects of the spin of the black hole, with the formation of giant higher order caustics and multiple images. Finally, we will consider the perspectives for observations of black hole lensing, from the

V. Bozza

Dipartimento di Fisica "E. R. Caianiello", Università di Salerno, I-84084 Fisciano, Italy. Istituto Nazionale di Fisica Nucleare, Sezione di Napoli, Italy.

Istituto Internazionale per gli Alti Studi Scientifici, I-84019, Vietri sul Mare, Italy. E-mail: valboz@sa.infn.it 
detection of secondary images of stellar sources to the interpretation of iron $\mathrm{K}-\alpha$ lines and direct imaging of the shadow of the black hole.

\section{Spherically Symmetric Black Holes}

The first presentation of the exact calculation of light deflection by a compact body using the Schwarzschild metric dates back to the work by Charles Darwin 1959 in 1 .

A generic static spherically symmetric metric can be put in the form

$$
d s^{2}=A(r) d t^{2}-B(r) d r^{2}-C(r)\left(d \vartheta^{2}+\sin ^{2} \vartheta d \phi^{2}\right) .
$$

In particular, the Schwarzschild metric has $A(r)=1-2 M / r, B(r)=[A(r)]^{-1}$, $C(r)=r^{2}$. Suppose that a photon comes from infinite distance, grazes the black hole at a minimum distance $r_{m}$ and goes away to infinity. The deflection angle, defined as the angle between the asymptotic incoming and outgoing trajectories, can be easily derived by the analysis of the geodesics equations [2]

$$
\alpha=-\pi+2 \int_{r_{m}}^{\infty} u \sqrt{\frac{B(r)}{C(r)\left[C(r) / A(r)-u^{2}\right]}} d r,
$$

where $u$ is the impact parameter, defined as the distance between the black hole and each of the asymptotic photon trajectories, related to the closest approach distance $r_{m}$ by

$$
u^{2}=\frac{C\left(r_{m}\right)}{A\left(r_{m}\right)} .
$$

The integral (2) was solved by Einstein assuming that $r \geq r_{m} \gg 2 M$. In this limit it reduces to the standard Einstein result for the Weak Deflection Limit (WDL)

$$
\alpha_{W D L}=4 M / u,
$$

with $u \simeq r_{m}$. Darwin, instead, made the integration exactly in the case of a Schwarzschild metric. His deflection angle is

$$
\alpha=-\pi+4 \sqrt{r_{m} / s} F(\varphi, m),
$$

where $F(\phi, m)$ is the elliptic integral of the first kind? and

$$
\begin{aligned}
& s=\sqrt{\left(r_{m}-2 M\right)\left(r_{m}+6 M\right)} \\
& m=\left(s-r_{m}+6 M\right) / 2 s \\
& \varphi=\arcsin \sqrt{2 s /\left(3 r_{m}-6 M+s\right)} .
\end{aligned}
$$

\footnotetext{
1 Charles Darwin was the grandson of the famous evolutionist with the same name, author of "The Origin of the Species".

2 In our notations $F(\varphi, m)=\int_{0}^{\varphi} \frac{d \theta}{\sqrt{1-m \sin ^{2} \theta}}$.
} 


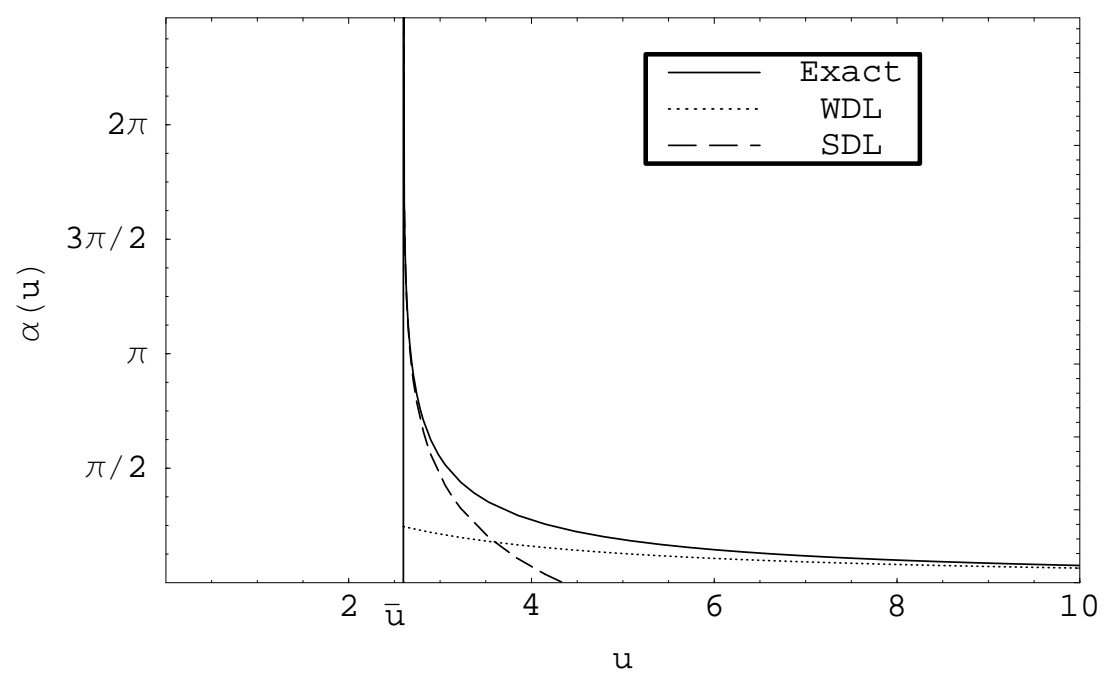

Fig. 1 Comparison between the exact deflection angle for photons in a Schwarzschild metric and the two limits discussed in the text. The impact parameter is in units of the Schwarzschild radius.

Eq. (5) gives the deflection angle as a function of the unobservable minimum distance $r_{m}$. We can better express it in terms of the impact parameter $u$ (which is coordinate-independent), using Eq. (3). The exact deflection angle is plotted in Figure 1] along with its approximations in the WDL (4), and the Strong Deflection Limit (SDL) to be introduced below (9).

Darwin's deflection angle and the Einstein approximation coincide at very large impact parameters. At small impact parameters, however, the salient feature of the exact deflection angle is the divergence at a finite value $u=\bar{u}=$ $3 \sqrt{3} M$, corresponding to a closest approach $r_{m}=\bar{r}=3 M$ via Eq. (3). Light rays with impact parameters closer and closer to $\bar{u}$ experience larger and larger deflections even exceeding $2 \pi$. This simply means that a light ray deflected with $\alpha$ in the interval $[2 n \pi, 2(n+1) \pi]$ performs $n$ complete loops around the black hole before leaving it. A light ray with impact parameter exactly equal to $\bar{u}$ would perform an infinite number of loops approaching $r=\bar{r}$ indefinitely. Nonetheless, circular orbits of light rays with radius $r=\bar{r}$ are admitted by geodesics equations, but are unstable against small perturbations, which would finally drive photons into the black hole or toward spatial infinity. $\bar{r}$ is also called the radius of the photon sphere (for a general definition see Ref. [3]). Finally, photons with impact parameters smaller than $\bar{u}$ are just captured by the black hole and fall into the horizon.

A good approximation for the deflection angle near the divergence is obtained by expanding the elliptic integral for $r_{m} \simeq \bar{r}$. We get

$$
\alpha_{S D L}=-\log (u / \bar{u}-1)+\log [216(7-4 \sqrt{3})]-\pi,
$$

which is the Strong Deflection Limit approximation plotted in Fig. 1 
Darwin himself intuited the possibility that a source lensed by a compact "star" with radius smaller than $\bar{r}$ would be replicated in two infinite sequences of images on each side of the len 3 . In fact, thanks to the divergence of $\alpha$, we can always find light rays with an impact parameter $u$ close enough to $\bar{u}$ so as to perform $n$ loops around the black hole (in either way) and finally reach the observer. These $n$-loop images or higher order images (called ghosts by Darwin and sometimes mirages or relativistic images) are missed in the WDL but can be well-described using the SDL approximation for the deflection angle.

In the following subsections we will discuss the black hole lens equation and derive the general features of the images. Finally we will review the main approximation schemes (WDL, SDL and non-perturbative).

\subsection{The Black Hole Lens equation}

A lens equation is a relation between the geometry (positions of source, observer and lens in a given coordinate system) and the position of the images in the observer's sky. Solving this equation, we can get the positions of the images as functions of the geometric parameters of the system.

The position of the observer and the final direction of the photon hitting the observer can be considered as initial conditions for the geodesics equations. Tracing them backward in the affine parameter, we can write down a formally exact lens equation 8 . This approach has been applied to the Schwarzschild lens 9 and then generalized to all spherically symmetric spacetimes 10] (see also [1]).

Let us consider a source at coordinates $4\left(r_{S}, \phi_{S}\right)$ and an observer at $\left(r_{O}, \phi_{O}\right)$, with $r_{O}>r_{S}$. In typical lensing situations, the photon is emitted from the source, approaches the black hole to a minimum distance $r_{m}$ and then escapes toward the observer. Therefore, we can divide the trajectory into an approach phase (from $r_{S}$ to $r_{m}$ with $\dot{5}<0$ ) and an escape phase (from $r_{m}$ to $r_{O}$ with $\dot{r}>0$ ). By simple integration of the geodesics equations, we can calculate the total azimuthal shift experienced by the photon as

$$
\Phi_{-}\left(u, r_{O}, r_{S}\right)=\left[\int_{r_{m}}^{r_{O}}+\int_{r_{m}}^{r_{S}}\right] u \sqrt{\frac{B(r)}{C(r)\left[C(r) / A(r)-u^{2}\right]}} d r .
$$

The two integrals separately cover the approach and escape phases of the trajectory. $u$ is the asymptotic impact parameter, related to $r_{m}$ by Eq. (3).

However, we must also consider the possibility that the photon directly goes from the source to the observer always moving away from the black hole

\footnotetext{
3 The existence of higher order images and/or the SDL expansion of Eq. (9) have been independently re-discovered several times [4,5,6,7, demonstrating how easy is to lose memory of old papers.

4 The spherical symmetry ensures that the motion takes place on a fixed plane. Choosing our coordinate system so that $\vartheta_{O}=\vartheta_{S}=\pi / 2$ we get $\vartheta=\pi / 2$ during the whole trajectory.

5 The dot denotes derivative with respect to the affine parameter.
} 


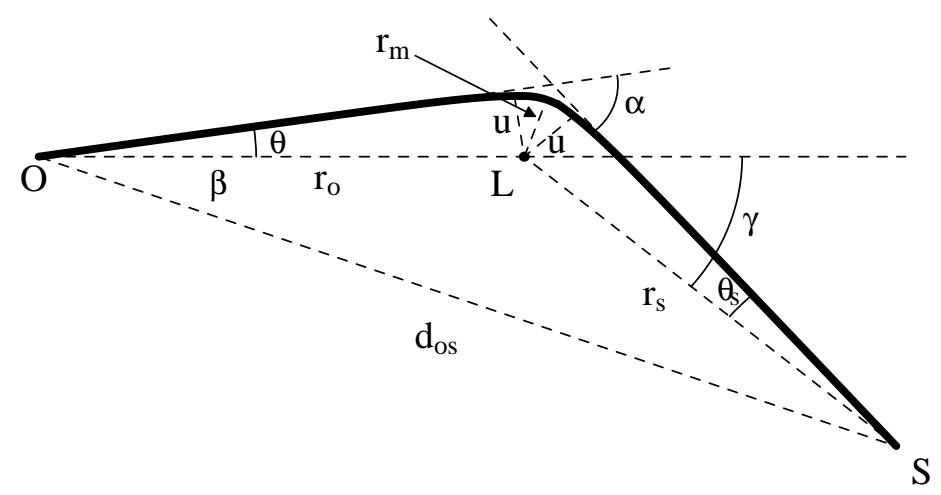

Fig. 2 Generic lensing geometry by a static spherically symmetric body $L$, illustrating the quantities defined in the text. The source is in $S$ and the observer in $O$.

with $\dot{r}>0$ (e.g. when the source is located between the lens and the observer). In this case, there is only an escape phase and the azimuthal shift is

$$
\Phi_{+}\left(u, r_{O}, r_{S}\right)=\int_{r_{S}}^{r_{O}} u \sqrt{\frac{B(r)}{C(r)\left[C(r) / A(r)-u^{2}\right]}} d r .
$$

Note that the two expressions coincide when $r_{S} \rightarrow r_{m}$, i.e. when the photon is emitted in a tangential direction $\dot{r}=0$.

The lens equation can be written by simply imposing that the azimuthal shift equals the difference in azimuth between source and observer modulo $2 \pi$.

$$
\Phi\left(u, r_{O}, r_{S}\right)=\Delta \phi \equiv \phi_{O}-\phi_{S}+2 n \pi
$$

where $\Phi=\Phi_{-}$if $\Delta \phi>\Phi_{ \pm}\left(u, r_{O}, r_{m}\right)$ and $\Phi=\Phi_{+}$otherwise. The integer number $n$ takes into account the fact that $\phi$ is a periodic coordinate.

The angle $\theta$ at which the observer detects the photon is 12

$$
\theta=\arcsin \left(u \sqrt{\frac{A\left(r_{O}\right)}{C\left(r_{O}\right)}}\right) .
$$

Once we fix the radial coordinates of source $r_{S}$ and observer $r_{O}$, and their relative azimuthal position $\Delta \phi$, by inverting the lens equation (12) we can get $u$ and thus the positions of the images $\theta$ through Eq. (13).

An interesting limit is obtained when source and observer are very far from the lens with respect to all other distance scales $(u$ and $M)$. Taking only the smallest powers in $u / r_{O}$ and $u / r_{S}$, we get the Ohanian lens equation [5]

$$
\alpha-\theta-\theta_{S}=-\gamma \equiv \Delta \phi-\pi,
$$



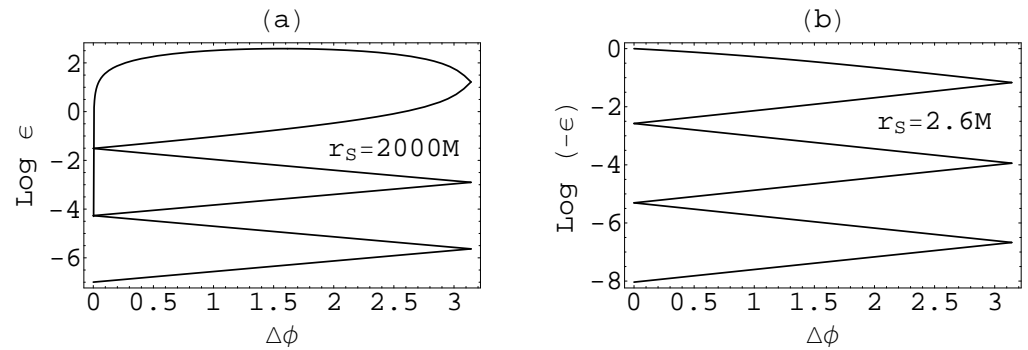

Fig. 3 Position of the images as a function of the source position $\Delta \phi$ for $r_{S}=2000 M$ (a) and $r_{S}=2.6 M(\mathrm{~b})$. In both cases $r_{O}=\infty . \epsilon \equiv(\theta / \bar{\theta}-1)$ is the relative distance of the image from the shadow border.

with $\sin \theta \simeq u / r_{O}, \sin \theta_{S}=u / r_{S}$ and $\alpha$ being the deflection angle between the asymptotic incoming and outgoing trajectories $(\alpha=\Phi(u, \infty, \infty)-\pi$, see Fig. 2). The Ohanian lens equation is an appealing simplification of Eq. (12), in that it gets rid of the two parameters $r_{O}$ and $r_{S}$. While the limit $r_{O} \simeq \infty$ is justified from the fact that the Earth is very far from any known candidate black hole, the limit $r_{S} \gg u$ leaves out the very interesting case in which the source is part of the environment of the black hole. A discussion on the errors committed when using the Ohanian or other approximate lens equations appeared in the literature is contained in Ref. [13].

\subsection{Position of the images}

Armed with our exact lens equation (12), we can now find the images for a generic source position varying from $\Delta \phi=0$ (source in front of the black hole) to $\Delta \phi=\pi$ (source behind the black hole). Only images on the same side of the source (direct images) can be obtained by Eq. (12), since we have assumed that $\dot{\phi}$ is always positive. However, thanks to the spherical symmetry, the position of the images on the opposite side of the source (indirect images) can be obtained by solving Eq. (12) with $\Delta \phi \rightarrow 2 \pi-\Delta \phi$. Thanks to the $2 n \pi$ on the right hand side of Eq. (12), we have one image in each interval $(m-1) \pi<\Phi<m \pi$ for any integer $m$. We will refer to this integer as the order of the image. The primary image will thus have $0<\Phi<\pi$, the secondary image $\pi<\Phi<2 \pi$ and so on for higher order images. Odd order images are direct and even order images are indirect.

In Fig. 3, we plot the position of the images as a function of $\Delta \phi$. The left panel, represents the case of a distant source $\left(r_{S}=2000 M\right)$, while the right panel considers a source very close to the black hole between the horizon $r=2 M$ and the photon sphere $r=\bar{r}=3 M$. The position of the images is expressed in terms of $\epsilon \equiv(\theta / \bar{\theta}-1)$, where $\bar{\theta} \equiv \bar{u} / r_{O}$ is called the shadow border for reasons that will be clear in a while. From top to bottom, the curves represent the primary image $(n=0)$, the secondary image $(\Delta \phi \rightarrow 2 \pi-\Delta \phi$, 
$n=0)$, the third order image $(n=1)$, the fourth order image $(\Delta \phi \rightarrow 2 \pi-\Delta \phi$, $n=1)$, the fifth and sixth order images $(n=2)$.

The familiar gravitational lensing geometry with the source behind the black hole is recovered at $\Delta \phi=\pi$. In case of perfect alignment, we expect the primary and secondary images to merge in the classical Einstein ring. Indeed, in Fig. 1a we see that they tend to the same value of $\epsilon$, that is to the same angle $\theta$ (but on opposite sides). As soon as the source moves out of perfect alignment $(\Delta \phi<\pi)$, the primary image moves farther from the black hole while the secondary image gets closer. For a source in quadrature $(\Delta \phi \simeq \pi / 2)$ the light bending is practically negligible for the direct image, so $\theta$ reaches the maximum value $r_{S} / r_{O}$ and then decreases to zero when $\Delta \phi=0$ (source in front of the black hole). On the other hand, the secondary image gets closer and closer to the black hole but always stays outside the circle of radius $\bar{\theta}$.

Coming to higher order images, we see that they also form Einstein rings when $\Delta \phi=\pi$, but with radius just very slightly larger than $\bar{\theta}$. As $\Delta \phi$ decreases, the direct images move farther and the indirect get closer to the shadow border. Without the logarithmic scale, all images would appear packed together at the shadow border $\bar{\theta}$. Finally, when the source is in front of the black hole $(\Delta \phi=0)$, every indirect image merges with the direct image of the next order to form an Einstein ring. Therefore, black holes surprisingly behave in a similar way with sources in front of the black hole and behind the black hole, since we can have an infinite sequence of concentric Einstein rings in both cases. The geometry with the source in front of the black hole is also known as retro-lensing [14,15. It is important to stress that in no case images of sources outside the photon sphere can appear inside the circle of radius $\bar{\theta}$, which justifies its name as shadow of the black hole.

Now let's move to Fig. 3b, which represents the same diagram for a source inside the photon sphere but outside the horizon. In this case, $\epsilon=\theta / \bar{\theta}-1$ is always negative, which means that the images form inside the shadow. The distance order of the images from the black hole is now reversed, with lower order images being closer to the black hole and higher order images appearing closer and closer to the border of the shadow. Apart from this major difference, the diagram of Fig. 3b is quite similar to 3a , with the formation of Einstein rings at $\Delta \phi=0, \pi$. Just note that at $\Delta \phi=0$ the direct image is again in $\theta=0$, corresponding to $\epsilon=-1$.

The dependence of the images on the source distance is illustrated in detail in Fig. 4. This figure shows how the radius of the Einstein rings (in standard lensing configuration $\Delta \phi=\pi$ ) depends on the source position. In Fig. 植 we have the primary Einstein ring and in Fig. 4b we have the second order Einstein ring. In both cases the rings enter the shadow when $r_{S}<3 M$. At very large $r_{S}$, however, the primary Einstein ring grows according to the WDL formula $\theta_{E}=\sqrt{4 M r_{S} / r_{O}\left(r_{O}+r_{S}\right)}$, whereas the higher order Einstein rings stay close to the shadow border. 
(a)

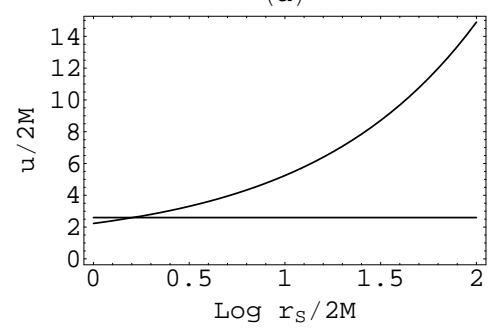

(b)

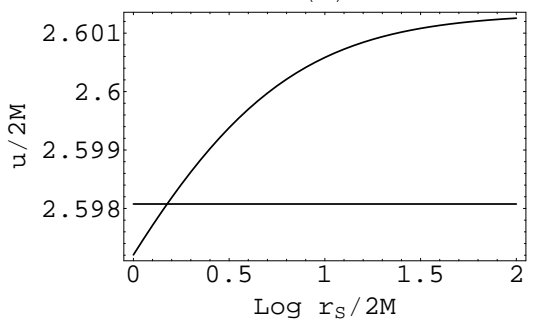

Fig. 4 Position of the Einstein ring vs source distance. (a) Primary ring, (b) Second order ring. The horizontal line represents the shadow border $\bar{u}=3 \sqrt{3} M$.

\subsection{Magnification}

The magnification of the images only makes sense for sources in the asymptotically flat region $r_{S} \gg M$. In fact, in order to compare angular elements of image and source, we need to project the source element to a Minkowski space. This process is coordinate-dependent unless the source is in the asymptotic region. Furthermore, the intensity of the image elements is also red-shifted if the source is deep in the gravitational field of the lens as a consequence of the conservation of the number of photons $I_{O} / \nu_{O}^{3}=I_{S} / \nu_{S}^{3}$.

Let us consider a source far from the black hole. An angular area element of the image has size $d u / r_{O}$ in the radial direction and $u d \eta / r_{O}$ in the tangential direction, where $\eta$ is an angular coordinate around the axis lens-observer. The corresponding tangential displacement in the source surface is $r_{S} \sin \Delta \phi d \eta$. The tangential magnification is therefore

$$
\mu_{t}=\frac{u / r_{O}}{r_{S} \sin \Delta \phi / d_{O S}}
$$

where

$$
d_{O S}=\sqrt{r_{O}^{2}+r_{S}^{2}-2 r_{O} r_{S} \cos \Delta \phi}
$$

is the (Euclidean) distance between source and observer 6 .

For the radial displacement the situation is more subtle. A shift in the impact parameter $d u$ selects a geodesic close to the original one. By differentiation of the lens equation (12), we get

$$
\frac{\partial \Phi}{\partial u} d u+\frac{\partial \Phi}{\partial r_{S}} d r_{S}=-d \phi_{S} .
$$

This equation approximates the perturbed geodesic in a neighbourhood of the source $\left(r_{S}, \phi_{S}\right)$. The point in which this geodesic will intercept a section orthogonal to the emission direction $\dot{x}_{e}^{i}$ is $\left(r_{S}, \phi_{S}\right)+d y^{i}$ with

$$
d y^{i} \equiv\left(d r_{S}, d \phi_{S}\right)=\left(u / r_{S}, \mp \sqrt{1-u^{2} / r_{S}^{2}} / r_{S}\right) d R,
$$

6 The Euclidean distance can be safely defined for sources and observers in the asymptotic region $r_{S}, r_{O} \gg M$, otherwise it loses meaning. 


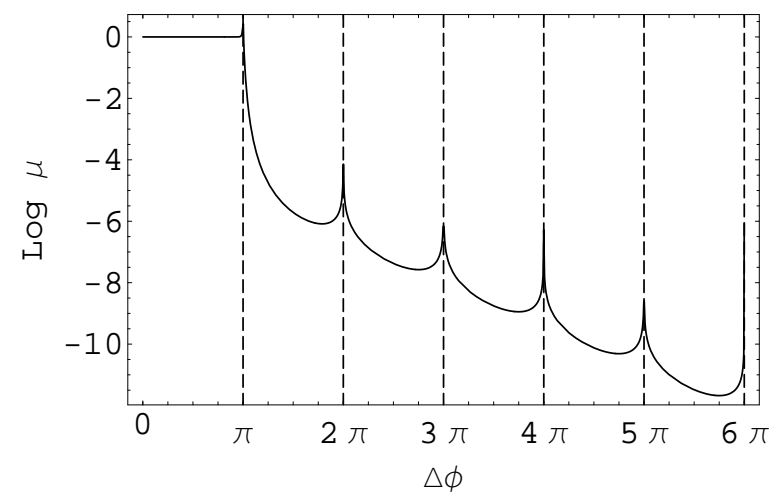

Fig. 5 Magnification of the images as a function of the azimuthal shift $\Delta \phi$ for the Schwarzschild lens.

which satisfies $7 \eta_{i j} \dot{x}_{e}^{i} d y^{j}=0$ and $\eta_{i j} d y^{i} d y^{j}=d R^{2}$. The distance $d R$ spans a source element. We can thus define the angular source element in absence of gravitational lensing as $d R / d_{O S}$. The double sign in $d \phi_{S}$ in Eq. (18) follows the sign of $\Phi_{ \pm}$that applies to the image under examination.

Finally, the radial magnification is

$$
\mu_{r}=\frac{d u / r_{O}}{d R / d_{O S}}
$$

where $d u$ and $d R$ are related by Eqs. (17) and (18). The total magnification is $\mu=\mu_{r} \mu_{t}$.

When $u \ll r_{S}$ and the photon is emitted with $\dot{r}<0$, our definition of magnification reduces to 5 .

$$
\left.\mu\right|_{r_{S} \rightarrow \infty}=\frac{d_{O S}^{2}}{r_{S}^{2}} \frac{\sin \theta}{\sin \Delta \phi} \frac{d \theta}{d \Delta \phi} .
$$

Fig. 5 shows the magnification as a function of the the azimuthal shift [5]. All images have been put in sequence by adding $(m-1) \pi$ to the abscissa of images of order $m$. We see that the magnification diverges at all multiples of $\pi$. This divergence comes from the tangential magnification (15), which means that images of a finite size-source become large arcs and then merge into rings when the source is in front or behind the black hole. The primary image $(0<\Delta \phi<\pi)$ has otherwise magnification very close to 1 , whereas all other images have an exponentially decreasing magnification. The secondary image interpolates between the two behaviours. We conclude that the lines defined by $\Delta \phi=0$ and $\Delta \phi=\pi$ and extending from the horizon to infinity $(2 M<r<\infty)$ represent degenerate (with zero section) caustic tubes for the Schwarzschild metric.

\footnotetext{
7 Here $\eta_{i j}=\operatorname{diag}\left(1, r^{2}, r^{2} \sin ^{2} \vartheta\right)$ is the Euclidean metric in polar coordinates as the source is in the asymptotic region.
} 
The parity of the images is determined by the factor $\sin \Delta \phi$ in the tangential magnification. All direct images have positive parity and all indirect images have negative parity.

We conclude by saying that possible effects beyond the geometrical optics scheme for photons travelling close to the photon sphere are still awaiting a full investigation. The existence of nonlinear dispersion relations for the photons propagating close to the photon sphere could have important consequences not yet considered [16].

\subsection{Analytical approximations}

The elliptic integrals of the exact treatment of light bending are not particularly illuminating and prevent an analytical inversion of the lens equation (12). For this reason, several approximation schemes have been developed in different limits. With their analytical formulae they shed new light on the plots of the previous subsection. Furthermore, taking advantage of the perturbative schemes in different limits, it is possible to compare different black hole metrics coming from alternative gravity theories and use gravitational lensing to discriminate among different scenarios.

\subsubsection{Weak deflection limit}

The WDL amounts to setting $u \gg M$ in the deflection integrals (5) or (10) and (11), which also implies $r_{m}, r_{S} \gg M$. We are thus in the lower-right corner of Fig. 1 or the upper side of Fig. 3 a. When $\Delta \phi$ is significantly less than $\pi$, the gravitational lensing effect on the primary image is negligible while the secondary image is not in the WDL regime. Therefore, the WDL approximation is only useful in the case $\Delta \phi \simeq \pi$, where it can be nicely employed to describe the primary and secondary images.

In the WDL it is possible to study a generic static spherically symmetric metric by using the PPN formalism. In isotropic coordinates, the metric can be parameterized as

$$
\begin{aligned}
& d s^{2}=A^{\prime}\left(r^{\prime}\right) d t^{2}-B^{\prime}\left(r^{\prime}\right)\left[d r^{\prime 2}+r^{\prime 2} d \Omega^{2}\right] \\
& A^{\prime}\left(r^{\prime}\right)=1-2 \alpha^{\prime} \frac{M}{r^{\prime}}+2 \beta^{\prime} \frac{M^{2}}{r^{\prime 2}}+\ldots \\
& B^{\prime}\left(r^{\prime}\right)=1+2 \gamma^{\prime} \frac{M}{r^{\prime}}+\frac{3}{2} \delta^{\prime} \frac{M^{2}}{r^{\prime 2}}+\ldots
\end{aligned}
$$

In the Schwarzschild limit, the PPN coefficients become $\alpha^{\prime}=\beta^{\prime}=\gamma^{\prime}=\delta^{\prime}=1$.

The integrals in the azimuthal shift (10) can be easily expanded in powers of $M$. In particular, the deflection angle (2) has been calculated to the second order in $M$ by Epstein and Shapiro already in 1980 [17] (see also [18]). Defining the Einstein angle

$$
\theta_{E}=\sqrt{4 M r_{S} /\left(r_{O} d_{O S}\right)}
$$


a convenient expansion parameter is 19

$$
\varepsilon=\theta_{E} d_{O S} /\left(4 r_{S}\right)
$$

Without gravitational lensing, the observer would see the source at angle $\beta$ from the lens. Simple geometry relates this angle to the azimuthal shift $\Delta \phi$ as $d_{O S} \sin \beta=r_{S} \sin \Delta \phi$. Setting $y \equiv \beta / \theta_{E}$, it turns out that to lowest order $\theta \sim$ $\theta_{E}$ and we can expand the position of the images as $\theta=\theta_{E}\left(\theta_{0}+\varepsilon \theta_{1}+\varepsilon^{2} \theta_{2}+\ldots\right)$.

Expanding the exact lens equation (12), we can collect all terms according to their order in $\varepsilon$. Finally, we can calculate the position of the images and their magnification $\mu=\left(\mu_{0}+\varepsilon \mu_{1}+\ldots\right)$ order by order. We just quote here the final result: 8 found by Ebina et al. 20] in the Schwarzchild case, by Sereno [21] for Reissner-Nordström and by Keeton and Petters for a generic PPN metric 19

$$
\begin{aligned}
& \theta_{0}=\left(y+\sqrt{y^{2}+A_{1}}\right) / 2, \quad \theta_{1}=A_{2} /\left(A_{1}+4 \theta_{0}^{2}\right) \\
& \mu_{0}=16 \theta_{0}^{4} /\left(16 \theta_{0}^{4}-A_{1}^{2}\right), \quad \mu_{1}=-16 \theta_{0}^{3} A_{2} /\left(A_{1}+4 \theta_{0}^{2}\right)^{3}
\end{aligned}
$$

with the coefficients $A_{1}$ and $A_{2}$ fixed by the PPN metric as

$$
A_{1}=2\left(\alpha^{\prime}+\gamma^{\prime}\right), \quad A_{2}=2 \alpha^{\prime 2}-\beta^{\prime}+2 \alpha^{\prime} \gamma^{\prime}+3 \delta^{\prime} / 4 \text {. }
$$

In principle, if the source is close enough to the lens so as to raise $\varepsilon$ significantly, by studying gravitational lensing we can gain access to the first order correction in the WDL expansion and thus infer the values of PPN parameters. Surprisingly, it turns out that all observables that are independent of the unknown source position are unchanged at first order in $\varepsilon$ [20] for all PPN metrics agreeing with General Relativity at zero order (thus having $\alpha^{\prime}+\gamma^{\prime}=2$ ) [19. This fact was prefigured by Damour and Esposito-Farèse [22] using an independent field theory approach to gravity theories in which they showed that $2 \mathrm{PN}$ parameters do not enter the equation of motion of light.

Finally, we note that the exact lens equation (12) is not really necessary to calculate the first order correction, as one would reach the same result using the classical small angles lens equation with a second order expansion of the deflection angle (2). The second order corrections in $\varepsilon$, however, can only be calculated by the exact lens equation or by the Ohanian lens equation (14) [23], as other lens equations would deviate from the correct results. The Ohanian lens equation, instead, would fail at the fourth order only 13 .

\subsubsection{Strong Deflection Limit}

The expansion of the elliptic integral in Darwin's formula at its divergence at $r=3 M$ leads to the SDL formula (9), which shows that the divergence at $u=3 \sqrt{3} M$ is logarithmic $1,7,14,24$. This is not a peculiarity of Schwarzschild metric, as even in Reissner-Nordström metric a similar divergence can be found

\footnotetext{
8 In these formulae $y$ is positive for the direct image and negative for the indirect image.
} 
25]. Actually, it is possible to prove that the logarithmic divergence in the deflection angle is a universal feature of all static spherically symmetric metrics endowed with a photon sphere $[26]^{9}$.

In order to calculate the SDL in a given metric, it is first necessary to calculate the radius of the photon sphere. This is obtained by imposing that the denominator of Eq. (2) has a double root at $r=r_{m}$. This is equivalent to the equation

$$
C^{\prime}(\bar{r}) / C(\bar{r})=A^{\prime}(\bar{r}) / A(\bar{r}),
$$

which yields the radius of the photon sphere $\bar{r}[3$. The minimum impact parameter $\bar{u}$ is then given by Eq. (3) with $r_{m}=\bar{r}$.

We can then expand the integrals in the azimuthal shift (10) in terms of $\epsilon=(u / \bar{u}-1)$ to get the SDL lens equation 10

$$
\Delta \phi+2 n \pi=-\bar{a} \log \left[\epsilon /\left(1-\bar{r} / r_{S}\right)\right]+\bar{b},
$$

where the coefficients $\bar{a}$ and $\bar{b}$ depend on the metric considered 11 and on the source radial coordinate $r_{S}$. Indeed, the SDL coefficients may vary in different alternative theories of gravity, representing a sort of identity card of the black hole metric and a possible key for discriminating between theories predicting deviations from General Relativity in strong fields [29,30]. In the Schwarzschild case

$$
\bar{a}=1, \quad \bar{b}=-2 \log \left[(3+\sqrt{3})\left(3+\sqrt{3+18 M / r_{S}}\right) /(36 \sqrt{6})\right] .
$$

Corrections to Eq. (30) at higher orders in $\epsilon$ have been worked out only in the Schwarzschild case for $r_{S} \rightarrow \infty$ 31.

This lens equation can be used to describe all images with $n \geq 1$, generated by photons looping at least once around the black hole (cfr. Fig. 3a). It becomes inaccurate only for the secondary image. It may even be used for sources inside the photon sphere (Fig. $3 \mathrm{~b}$ ). The position of the images is found by inverting Eq. (31) and remembering that $\theta=u / r_{O}$ in the limit $r_{O} \gg M$. We have 28]

$$
\theta_{n}=\bar{\theta}\left[1+\left(1-\bar{r} / r_{S}\right) e^{(\bar{b}-\Delta \phi-2 n \pi) / \bar{a}}\right] .
$$

The indirect images are found by replacing $\Delta \phi$ by $2 \pi-\Delta \phi$ as usual. This formula indicates that the higher order images tend to the shadow border $\bar{\theta}$ with an exponential law (which justifies our choice of the logarithmic scale in Fig. (3). The images form outside the border for $r_{S}>\bar{r}$ and inside it otherwise.

\footnotetext{
${ }^{9}$ It is easy to prove that all static spherically symmetric asymptotically flat metrics with an event horizon have a photon sphere.

10 The SDL was developed in Ref. 26] for standard lensing only. The extension to the retrolensing configuration was done in Ref. [27. Here we present the most general treatment of Ref. 28.

11 They can be calculated analytically or numerically using the procedure described in Ref. [26] for sources at infinity and in Ref. 28, for any source positions.
} 
For sources very far from the black hole, the magnification is [5, 26$]$

$$
\mu_{n}=\left(\frac{D_{O S}}{r_{S}}\right)^{2} \frac{\bar{\theta}^{2}}{\bar{a} \sin \Delta \phi} e^{(\bar{b}-\Delta \phi-2 n \pi) / \bar{a}}
$$

which nicely reproduces the behavior of Fig. 5 and also proves that higher order images are exponentially fainter and fainter.

It is interesting to note that the time delay between two consecutive higher order images is approximately $\Delta T=2 \pi \bar{\theta} r_{O}$, which could be used in principle to infer the mass and distance of the black hole 32 .

Another interesting application of the SDL scheme is in the study of a static spherically symmetric black hole embedded in an external gravitational field. In the case of the standard WDL, Chang and Refsdal [33] showed that the zero order Einstein ring becomes elliptical and the corresponding degenerate caustic tube at $\Delta \phi=\pi$ acquires a diamond-shaped cross-section with angular size proportional to the external shear $\gamma$ and $\theta_{E}$. In the SDL, a correct description of the tidal forces 34 ] leads to the conclusion that also higher order Einstein rings gets slightly distorted and their corresponding caustic tubes are diamondshaped with size proportional to $\gamma \bar{\theta}[35$.

\subsubsection{Non-perturbative methods}

While the WDL describes the two main images in the best alignment regime between source and lens $(\Delta \phi \simeq \pi)$ and the SDL describes all higher order images $(n>0)$, no perturbative approximations are possible for the secondary image when the source is not aligned behind the black hole $(n=0$ and $0<$ $\Delta \phi<\pi)$. Indeed Figs. 3 and 5 show that the secondary image interpolates between the WDL and the SDL regimes when $\Delta \phi$ runs from $\pi$ to 0 , going from $\theta \sim \theta_{E}$ to $\theta \sim \bar{\theta}$.

A nice strategy to obtain simple analytic formulae has been proposed by Amore and collaborators [36]. First, the integral (2) is written in the form

$$
\alpha=2 \int_{0}^{1} \frac{d z}{\sqrt{V(1)-V(z)}}-\pi
$$

with $z=r_{m} / r$ and

$$
V(z)=z^{2} C(r) / B(r)-C^{2}(r) A\left(r_{m}\right) /\left[B(r) A(r) C\left(r_{m}\right)\right]+A\left(r_{m}\right) / C\left(r_{m}\right)
$$

being a sort of potentia 12 in the variable $z$.

Then, the potential $V(z)$ is replaced by a simpler form $V_{0}$ and the integral becomes

$$
\begin{aligned}
& \alpha=2 \int_{0}^{1} \frac{d z}{\sqrt{V_{0}(1)-V_{0}(z)}} \frac{1}{\sqrt{1+\delta \Delta(z)}}-\pi \\
& \Delta(z)=\frac{V(1)-V(z)}{V_{0}(1)-V_{0}(z)}-1 .
\end{aligned}
$$

12 Interestingly, the lensing problem is reformulated as an oscillator problem. 
Finally, the integral is solved as a series in powers of $\delta$ and calculated in $\delta=1$ (where Eq. (36) coincides with (34)). The convergence of the series depends on the chosen form of the auxiliary potential $V_{0}$. If $V_{0}$ depends on some shape parameter $\lambda$, this can be fixed by requiring the minimum sensitivity of the deflection angle (as in a variational method).

As a practical example, taking $V_{0}=\lambda z^{2}$ in the Schwarzschild metric, Amore and Arceo [36] obtained

$$
\Delta \phi=\pi\left(\frac{1}{\sqrt{1-8 M /\left(\pi r_{m}\right)}}-1\right),
$$

which works nicely in the intermediate regime for the zero order indirect image. More sophisticated potentials may lead to even better approximations englobing the main features of the WDL and SDL [36] at the price of dealing with more involved formulae.

\section{Rotating black holes}

Most astrophysical systems are known to have non-negligible angular momentum. Therefore it is natural to expect that physical black holes should be properly described by the Kerr metric. The study of null geodesics in Kerr geometry has been initiated by Carter in 1968 [37], who separated the Hamilton-Jacobi equation and wrote the geodesics equations in terms of first integrals of the motion

$$
\begin{gathered}
\pm \int \frac{d r}{\sqrt{R}}= \pm \int \frac{d \vartheta}{\sqrt{\Theta}} \\
\phi_{f}-\phi_{i}=a \int \frac{r^{2}+a^{2}-a J}{\Delta \sqrt{R}} d r-a \int \frac{d r}{\sqrt{R}}+J \int \frac{\csc ^{2} \vartheta}{\sqrt{\Theta}} d \vartheta
\end{gathered}
$$

where

$$
\begin{aligned}
& \Theta=Q+a^{2} \cos ^{2} \vartheta-J^{2} \cot ^{2} \vartheta ; \Delta=r^{2}-2 M r+a^{2} \\
& R=r^{4}+\left(a^{2}-J^{2}-Q\right) r^{2}+2 M\left(Q+(J-a)^{2}\right) r-a^{2} Q,
\end{aligned}
$$

$J$ and $Q$ are constants of motion labelling the geodesic under examination $13, a$ is the angular momentum of the black hole divided by its mass and expressed in natural units. The Kerr metric describes a rotating black hole if $a<M$ with horizon at $r_{H}=M+\sqrt{M^{2}-a^{2}}$, otherwise it represents a naked singularity. Both $\dot{r}$ and $\dot{\vartheta}$ may change sign during the motion. Therefore, every integral should be calculated from the emission point to the first inversion point (if any), then between each pair of consecutive inversion points, up to the final observation point. In the following, we will use the notation $\mu=\cos \vartheta 14$.

\footnotetext{
$13 \mathrm{~J}$ is the projection of the angular momentum of the photon on the rotation axis, $Q$ is a combination of the square of the other components of the angular momentum of the photon with the black hole angular momentum.

14 Not to be confused with the magnification $\mu$ of the previous section.
} 
The main difference with respect to spherically symmetric black holes is that the motion of the photon is no longer confined on a single plane, as the angular momentum of the black hole induces an orbital precession around its rotation axis. As a consequence, the polar angle $\vartheta$ cannot be eliminated by a simple choice of coordinates and the problem is fully three-dimensional. In principle, all integrals can be written in terms of elliptic integrals (see e.g. [38]). This is not particularly illuminating for the analytic point of view, but may be useful for implementing numerical codes for Kerr-geodesics tracing 39 , 40, 41,42. In particular, the analytic deflection angle for photons confined in the equatorial plane takes a reasonably short expression [43].

A thorough presentation of the classification and the properties of Kerr geodesics is in Ref. [24]. In order to investigate the rotation of the polarization plane of electromagnetic radiation (not discussed in this text), it is useful to introduce a tetrad formalism 44. As for spherically symmetric case, several studies of Kerr lensing in the WDL [17,45, 46, 47] and in the SDL [28, 48, 49 have been carried out. These studies allow to get interesting analytical approximations in some limits that we will sometimes recall later.

In the following subsections, we will give a sketch of the main characteristics of lensing by rotating black holes. We will first describe the shadow of the black hole, then we will discuss the caustics and finally the formation of the images.

\subsection{Shadow of the Kerr black hole}

Null geodesics are fully identified by the constants of motion $J$ and $Q$. For a static observer in the asymptotic flat space $\left(r_{O} \gg M\right)$, the position in the sky in which a photon is detected is completely determined by the values of these constants. In fact, defining $\mu_{O}=\cos \vartheta_{O}$, we have

$$
\begin{aligned}
& \theta_{1}=-J\left(r_{O} \sqrt{1-\mu_{O}^{2}}\right)^{-1}, \\
& \theta_{2}= \pm r_{O}^{-1} \sqrt{Q+\mu_{O}^{2}\left[a^{2}-J^{2} /\left(1-\mu_{O}^{2}\right)\right]}
\end{aligned}
$$

where $\theta_{1}$ is the angular distance from the black hole center projected orthogonally to the spin direction and $\theta_{2}$ is the same angular distance projected along the projection on the spin on the sky. The problem of determining the position of the images of a given source can thus be reformulated in terms of $J$ and $Q$.

Similarly to the spherically symmetric case, the integrals in the radial coordinate diverge logarithmically if the argument of the square root has a double root. This happens when $R$ and $\partial R / \partial r$ simultaneously vanish at the same point $\bar{r}$. The two equations $R=0$ and $R^{\prime}=0$ can be solved in terms of the two constants of motion $J$ and $Q$ as functions of $\bar{r}$, so as to find those geodesics 


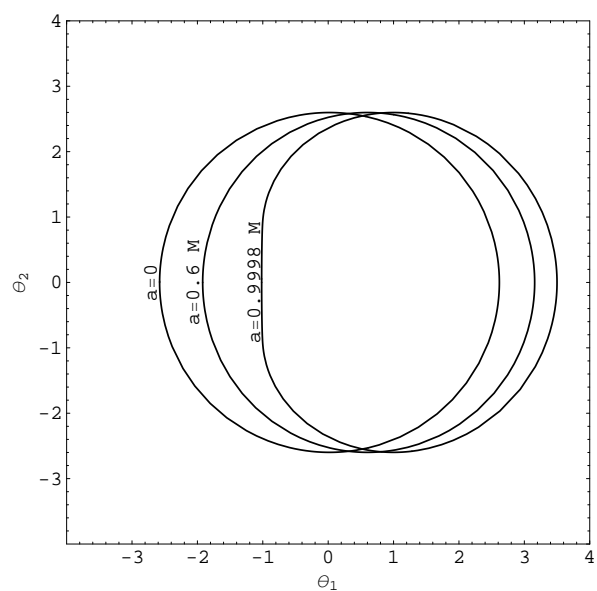

Fig. 6 The shadow border as seen by an equatorial observer for different values of the black hole spin. $\theta_{1}$ and $\theta_{2}$ are the angular coordinates in the observer's sky in units of $2 M / r_{O}$. The black hole is in the origin and the spin axis points in the positive $\theta_{2}$ direction.

which asymptotically approach an orbit at a fixed radius $\bar{r}$. Explicitly, we have

$$
\begin{aligned}
& \bar{J}(\bar{r})=\frac{\bar{r}^{2}(\bar{r}-3 M)+a^{2}(M+\bar{r})}{4 M^{2} a(M-\bar{r})} \\
& \bar{Q}(\bar{r})=\frac{\bar{r}^{3}\left[16 M^{3} a^{2}-\bar{r}(\bar{r}-3 M)^{2}\right]}{16 M^{4} a^{2}(\bar{r}-M)^{2}}
\end{aligned}
$$

which describe a locus in the $(J, Q)$ space parameterized by $\bar{r}$. Only positive values of $Q$ describe geodesics approaching infinity (see Eq. (44)). This constrains the possible values of $\bar{r}$ into a finite interval $\left[\bar{r}_{+}, \bar{r}_{-}\right]$defined by the condition $15 \geq 0$. The two extreme values $\bar{r}_{ \pm}$lead to a vanishing $Q$, which corresponds to a photon moving on an equatorial orbit. The lower value $\bar{r}_{+}$ corresponds to a photon moving with positive $J$, i.e. rotating in the same sense with the black hole (prograde photon). The higher value $\bar{r}_{-}$gives a negative $J$, thus describing a photon counter-rotating with respect to the black hole (retrograde photon). In-between, we can have orbits with any possible inclinations.

From the point of view of the observer at infinity, thanks to Eqs. (43) and (44), all geodesics asymptotically starting in an orbit at fixed radius $\bar{r}$, described by Eqs. (45) and (46), also describe a locus in the sky coordinates $\left(\theta_{1}, \theta_{2}\right)$. Notice that if the observer is not on the equator, equatorial orbits will never reach it. Indeed, the range of values of $\bar{r}$ that describe orbits ending at the observer is defined by the condition that the argument of the square root in Eq. (44) is positive. The new range $\left[\bar{r}_{<}, \bar{r}_{>}\right]$is obviously smaller than $\left[\bar{r}_{+}, \bar{r}_{-}\right]$and coincides with it if the observer is on the equator.

\footnotetext{
15 The third degree equation $Q=0$ has always two roots outside the horizon.
} 
Analogously to spherically symmetric black holes, the locus $\left(\bar{\theta}_{1}(\bar{r}), \bar{\theta}_{2}(\bar{r})\right)$ is called the shadow border of the Kerr black hole. In Fig. 6 we show the shadow border for three values of the spin parameter $a$. Indeed, when $a \rightarrow 0$, the shadow becomes a circle with radius $3 \sqrt{3} M / r_{O}$ as for the Schwarzschild black hole. When we turn the spin on, the shadow shifts to the right (the spin direction points to the top in Fig. 6) and becomes more compressed. With our coordinate system, photons detected on the left side of the black hole have rotated in the same sense of the black hole whereas photons detected on the right side have counter-rotated. As mentioned before, prograde photons are allowed to get closer to the black hole (up to $\bar{r}_{+}$) and therefore reach the observer with a small angular separation from the black hole. In the extremal limit $a \rightarrow M$, the shadow border on the left (prograde) side is flattened while $\bar{r}_{+}$equals the horizon radius $r_{H}$. Beyond this limit, the horizon disappears and the Kerr metric describes a naked singularity whose appearance is no longer determined by the last circular orbits of the photons but depends on the physical interpretation of the singularity itself 50 .

The idea of measuring the spin and other characteristics of astrophysical black holes from the shape of the shadow border dates back to Bardeen in 1972 [51] and has been re-proposed several times [24,50,52]. However, the real appearance of an astrophysical black hole is largely dictated by the properties of the accretion flow, as we shall discuss in the next section.

\subsection{Caustics}

As customary in gravitational lensing, the general functioning of a lens model is determined by its caustic structure. In fact, by studying caustics, we can find the points in which a source is maximally amplified and the regions in which new pairs of images are created. As showed in the previous section, spherically symmetric black holes have degenerate caustics in the form of zero-size tubes lying on the observer-lens axis behind and in front of the black hole. It can be easily imagined that the loss of spherical symmetry makes these tubes shift and acquire a finite extension. The existence of finite-size caustics in the Kerr metric was pointed in 1972 by Cunningham and Bardeen [53, who studied gravitational lensing of a star orbiting a Kerr black hole. They found that additional pairs of images were created when the source was in some particular regions. The complete shape of the primary caustic was instead illustrated for the first time by Rauch and Blandford [38. who showed that it becomes a finite-size tube with astroid cross-section, winding around the horizon. Lately, we discussed the complete caustic structure of the Kerr metric, illustrating higher order caustics and their dependence on spin and the observer position [54].

In order to calculate caustics, it is necessary to put Eqs. (39) and (40) in the form of a lens mapping relating the source coordinate: $16\left(\vartheta_{S}, \phi_{S}\right)$ to the

\footnotetext{
16 The source radial coordinate $r_{S}$ is considered as a parameter. For each value of $r_{S}$ we then obtain a section of the caustic tube at fixed distance from the black hole.
} 
image position $\left(\theta_{1}, \theta_{2}\right)$. After that, we have to calculate the Jacobian determinant and find the locus defined by its vanishing (critical curve). Finally, the critical curve is mapped to the source coordinates through the lens mapping to obtain the caustic. Everything can be done numerically, but the details are quite cumbersome and are summarized very shortly in this treatment. On the other hand, some analytic formulae can be obtained in the WDL and SDL analogously to what happens in spherically symmetric black holes.

In brief, it is convenient to replace the coordinates in the observer's sky $\left(\theta_{1}, \theta_{2}\right)$ by pseudo-polar coordinates centered on the black hole position. Fixing the angular coordinate $\eta$, the angular distance $\epsilon$ from the shadow, defined in analogy to what is done in the SDL, determines how much the photon is deflected from the black hole. In fact, a photon detected far from the black hole $(\epsilon \gg 1)$ is weakly deflected, whereas the deflection diverges when the photon is detected by the observer infinitely close to the shadow $(\epsilon \rightarrow 0)$. The number of oscillations in the polar angle $\vartheta$ depends on the radial coordinate $\epsilon$. It turns out that in the range $\left[\epsilon_{m}, \epsilon_{m+1}\right]$ in which the photon performs $m$ inversions in the polar motion, there is only one point in which the Jacobian determinant vanishes. Therefore, we can identify the caustic order with the number of inversions in the polar motion of photons that form the corresponding critical images. The primary caustic is formed by photons experiencing only one inversion in the polar motion. The secondary caustic is formed by photons performing two inversions in the polar motion and so on.

Fig. [7 is a 3 -dimensional representation of the primary caustic [38 for a quasi-extremal Kerr black hole and Fig. $7 \mathrm{~b}$ shows its cross-section at various distances. We can see that the zero-size tube of the Schwarzschild black hole here becomes a tube with astroid cross-section winding clockwise around the horizon an infinite number of times [38,54. At large distances $\left(r_{S} \gg M\right)$, the caustic can be described analytically in the WDL, since the photons of the critical images are weakly deflected. As shown by Sereno and De Luca 47], the primary caustic can be written in the coordinates $\left(\mu_{S}=\cos \vartheta_{S}, \phi_{S}\right)$ as

$$
\begin{aligned}
\phi_{S} & =-\pi-4 a_{m} \varepsilon^{2}-\frac{5}{4} \pi a_{m} \varepsilon^{3} \\
& +\left[\left(\frac{225}{128} \pi^{2}-16\right) a_{m}-\frac{15}{16} \pi a_{m}^{2} \sqrt{1-\mu_{O}^{2}} \cos ^{3} \eta\right] \varepsilon^{4} \\
\mu_{S} & =-\mu_{O}-\frac{15}{16} \pi a_{m}^{2}\left(1-\mu_{O}^{2}\right)^{3 / 2} \varepsilon^{4} \sin ^{3} \eta,
\end{aligned}
$$

with $a_{m}=a / M, \eta$ ranging from $-\pi$ to $\pi$ and $\varepsilon$ being the WDL expansion parameter already introduced in spherically symmetric black holes in Eq. (25). It is interesting to note that the caustic remains infinitely thin at second and third order in $\varepsilon$. We need to push the expansion to the fourth order in order to find a finite extension with the typical astroid cross-section. This fact signals that to lowest order the Kerr black hole can be perfectly mimicked by a Schwarzschild black hole with a finite shift, as previously noted by Asada and Kasai 46. The angular size of the primary caustic varies with distance as $\varepsilon^{4} \sim r_{S}^{-2}$. Therefore its physical extension shrinks as $r_{S}^{-1}$ far from the black 
( )

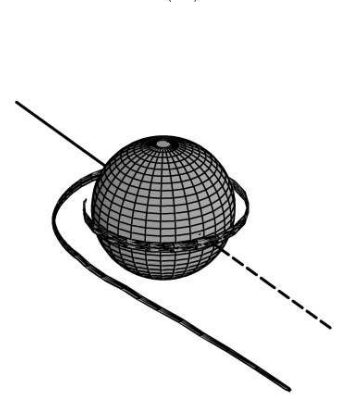

(c)

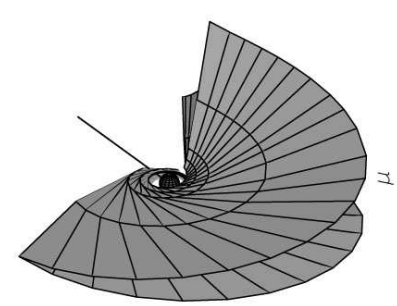

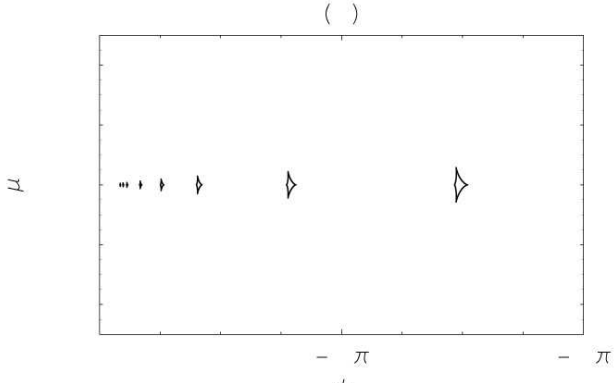

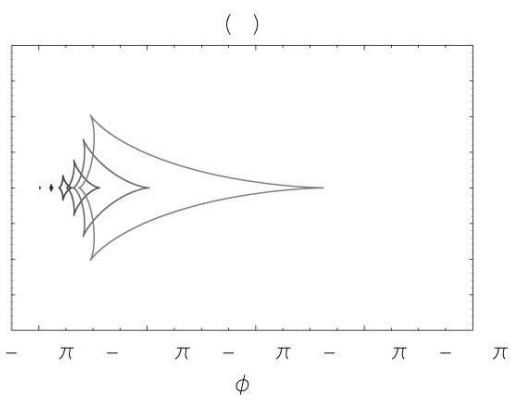

Fig. 7 (a) The primary caustic tube for $a=0.9998 M$ and $\mu_{O}=0$ in a 3-dimensional representation, with pseudo-euclidean coordinates $x=r \sin \vartheta \cos \phi, y=r \sin \vartheta \sin \phi, z=r \cos \vartheta$. The spin axis is directed toward the top. The straight solid line indicates the direction towards the observer, whereas the dashed line points in the opposite direction. The primary caustic for a Schwarzschild black hole $(a=0)$ coincides with this dashed line. (b) Cross section of the same primary caustic at various distances. From left to right, the radial coordinate is $r=5 M, 4.5 M, 4 M, 3 M, 2.3 M, 1.82 M, 1.42 M, 1.22 M$. (c) 3-dimensional picture of the second order caustic surface of an extremal Kerr black hole for an observer on the equatorial plane in the direction indicated by the solid line. The surface has been plotted for radial distances in the range $[2.2 M, 20 M]$. (d) Second order asymptotic caustic for an equatorial observer $\mu_{o}=0$ and different values of the spin. From left to right, $a=0.02 M, 0.2 M, 0.4 M, 0.6 M, 0.8 M, 0.9998 M$.

hole, severely limiting chances of observations of spin effects in the WDL. The effects of the spin becomes sizeable at stronger deflections, which can be obtained either with sources at distances from the black hole comparable with the Schwarzschild radius or considering higher order images.

The secondary caustic involves photons with two inversions in the polar motion. In the Schwarzschild limit it reduces to an infinitely thin tube on the same side of the observer. Its corresponding critical curve is the first retrolensing Einstein ring. In Fig. 7fd we can see what happens when we turn the spin on [54. The secondary caustic becomes very large and for quasi-extremal spins has an angular extension larger than $\pi$ in the azimuthal direction. Fur- 
thermore, the angular size does not decrease with the distance $r_{S}$, and the whole cross-section tends to an asymptotic astroid shape that is what we show in $7 \mathrm{~d}$. An impressive 3 -dimensional view of the secondary caustic in the quasi-extremal case is shown in Fig. 75. The caustic surface includes most of the equatorial plane assuming a discoidal shape. Creation and destruction of images is thus much more common in the second Einstein ring, than in the primary one. The spin effects, which are crucial to determine the size and the position of the secondary caustic, are then much more evident on higher order images, but we must be aware that these are much fainter than the primary images 17 . Also for higher order caustics it is possible to derive analytical approximations in a specific limit (SDL and small values of the spin, with $r_{S}$ free to vary from $r_{H}$ to infinity) [28, 49]. In this case for a caustic of order $m \geq 2$ we have

$$
\begin{aligned}
& \mu_{S}=(-1)^{m}\left[\mu_{O}+R_{m}\left(1-\mu_{o}^{2}\right)^{3 / 2} \sin ^{3} \eta\right], \\
& \phi_{S}=-m \pi-\Delta \phi_{m}+R_{m} \sqrt{1-\mu_{O}^{2}} \cos ^{3} \eta
\end{aligned}
$$

where the azimuthal shift is

$$
\Delta \phi_{m}=-a\left\{\frac{2 m \pi}{3 \sqrt{3}}+2 \log (2 \sqrt{3}-3)+\log \left[\frac{\left(2 \sqrt{r_{S}}+\sqrt{6 M+r_{S}}\right)}{3 \sqrt{\left(r_{S}-2 M\right)}}\right]\right\}
$$

and the semi-amplitude of the caustic is

$$
R_{m}=a^{2}\left[\frac{1}{18}(5 m \pi+8 \sqrt{3}-36)+\frac{\left(9 M+2 r_{S}-2 \sqrt{r_{S}} \sqrt{6 M+r_{S}}\right)}{3 \sqrt{3} \sqrt{r_{S}} \sqrt{6 M+r_{S}}}\right] .
$$

As for the primary caustic, the shift is linear and the size is quadratic in the spin. It should be noted that for small values of the spin there is a degeneracy 18 between the spin $a$ and the latitude of the observer on the equatorial plane. The caustics become smaller and smaller as the observer is moved from the equatorial plane to the polar axis, finally returning to zero shift and size when the observer is perfectly aligned with the rotation axis restoring the axial symmetry. The degeneracy between spin and observer latitude is only broken at high values of the spin [54].

\subsection{Formation of the images}

The discussion of the images in Kerr black hole lensing may start from the Schwarzschild limit. In this limit, we know that we have an infinite sequence of direct images and an infinite sequence of indirect images. We have noticed that

\footnotetext{
17 Fig. 5 in the Schwarzschild case may be considered as a good reference even in the Kerr case for a rough estimate of the luminosity of images of various orders.

18 The degeneracy is evident when switching to coordinates centered on the zero-order caustic position $\mu=(-1)^{m} \mu_{O}$. Then all quantities depend on the combination $a \sqrt{1-\mu_{O}^{2}}$.
} 
the image of order $m$ has an azimuthal shift $\Delta \phi$ in the range $[(m-1) \pi, m \pi]$. In the Kerr case, it is possible to maintain this identification using the number of oscillations in the polar angle, instead [53].

Solving Eq. (39) explicitly in $\mu_{S}$, we have

$$
\mu_{S}= \pm \mu_{+} \operatorname{sn}(2 K(k) \psi, k),
$$

where $\mu_{+}, k$ and $\psi$ are functions of $J$ and $Q, K(k)=F(\pi / 2, k)$ is the complete elliptic integral of the first kind and sn is the Jacobi elliptic function. As sn has period $4 K(k), \psi$ counts the number of half-oscillations on the equatorial plane. It turns out that there is always at least one image in each interval $m-1<\psi<m$. More specifically, when the source is inside the caustic of order $m$, there are three images, otherwise only one.

Comparing with the Schwarzschild black hole, in that case we had an image in each interval $(m-1) \pi<\Delta \phi<m \pi$. Direct images were those with odd $m$ and indirect images were those with even $m$. In the Kerr black hole the situation is similar, but the azimuthal shift is no longer suitable to label them, because it evolves differently for photons with different inclinations on the equatorial plane. The argument $\psi$ of the sn function in Eq. (53) provides the correct variable. Its explicit expression is reported in Ref. 54.

In Fig. 8 we show the position of the images of order 3 and 4 for a source very close to the caustic of order 3 , which involves photons performing a bit more than one loop and a half $(|\phi| \gtrsim 3 \pi)$. When the source is outside the caustic (Fig 8 a), there is one third order image (on the left) and one fourth order image (on the bottom right). The images appear as very stretched thin arcs. When the source is inside the caustic, two more third order images appear on the top right, while the fourth order image remains lonely.

The Kerr lens equation for sources close to caustics assumes a very simple form for small values of the spin. In this limit, we have [30,49,47.

$$
\delta \vartheta_{s} \cos \eta+(-1)^{m}\left(\delta \phi_{s}-R_{m} \cos \eta\right) \sin \eta=0,
$$

where $\left(\delta \vartheta_{s}, \delta \phi_{s}\right)$ is the source position relative to the center of the caustic, $R_{m}$ is the caustic radius 19 , and $\eta$ is the angular coordinate around the shadow border in the observer's sky as defined in Section 3.2. The distance from the shadow $\epsilon$ remains the same as in Schwarzschild to lowest order in $a$.

For near-extremal black holes $(a \rightarrow M)$, the azimuthal size of higher order caustics exceeds $2 \pi$ 54. As $\phi$ is a periodic coordinate, this means that we should consider the possibility that a source crosses the same caustic surface at different number of loops. When this happens, new pairs of additional images appear at the same order $m$ but with $\Delta \phi$ differing by multiples of $2 \pi$. From the physical point of view, this means that these pairs of images are formed by photons performing the same number of oscillations $m$ in the polar angle but a different number of loops in azimuth $\phi$. These new pairs of images appear on the flattened side on the left of the shadow and are formed by prograde photons moving very close to the equatorial plane. As the extension of higher

19 It can be obtained from Eqs. (47) and (48) for $m=1$ and from Eq. (52) for $m>1$. 
(a)

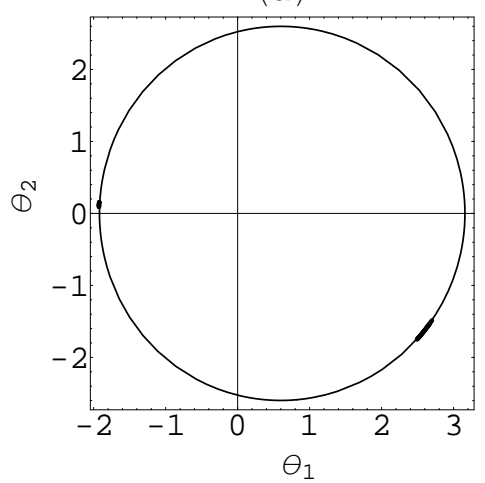

(b)

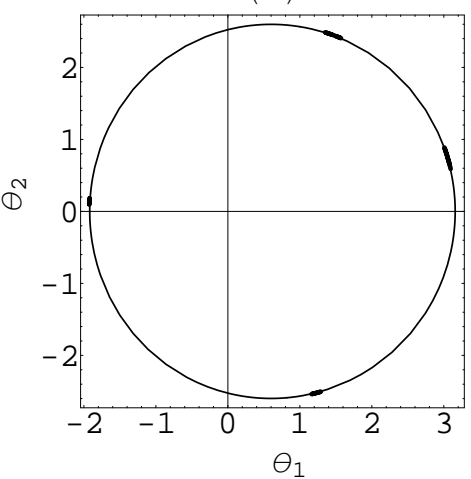

(c)

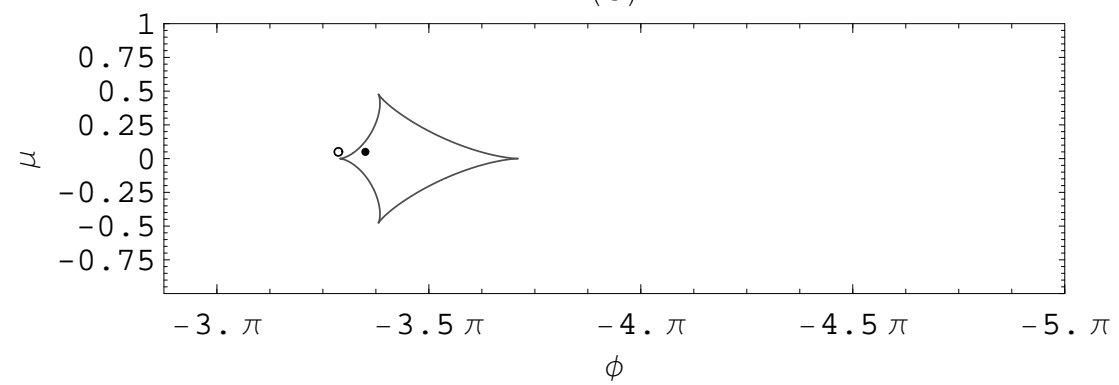

Fig. 8 Images of order 3 and 4 of a source of radius $2 M$ at $r_{S}=200 M$ seen by an observer on the equatorial plane of a black hole with spin $a=0.6 M$. In panel (c) we show two positions of the source with respect to the third order caustic. In panel (a) we show the images corresponding to the empty circle along with the shadow border and in panel (b) we show the images corresponding to the filled circle.

order caustics increases exponentially with the order $m$, the number of images also grows exponentially with $m$ in extremal Kerr black holes. However, the magnification drops even faster, so that it will be extremely difficult to observe this effect.

\section{Observational perspectives}

Black holes offer a possibility (unique in the astrophysical panorama) of studying light bending beyond the first order WDL approximation, which governs all known cases of gravitational lensing. However, unveiling this new phenomenology requires very powerful technical capability. Let us start with a general discussion. 
The first corrections to the primary and secondary images, which are wellknown in all standard gravitational lensing situations, scale with the combination $\theta_{E} \varepsilon$, which is proportional to $M / r_{O}$. Interestingly, also the angular size of the shadow of the black hole and all observables related to higher order images are proportional to the ratio $M / r_{O}$. We shall therefore take the shadow angular size $\bar{\theta}=3 \sqrt{3} M / r_{O}$ as the guiding quantity to evaluate astrophysical black holes as candidates for gravitational lensing beyond the WDL standard approximation.

Typical stellar black holes in the Galaxy have

$$
\bar{\theta}_{\text {Stellar }}=5.1 \times 10^{-11} \operatorname{arcsec}\left(\frac{M}{M_{\odot}}\right)\left(\frac{r_{O}}{1 \mathrm{kpc}}\right)^{-1}
$$

which is definitely a too small angle to be observed. Much promising candidates are massive black holes at the center of galaxies. In particular, the black hole in the center of the Milky Way, identified with the radio source Sgr A* 55, has mass $4.31 \times 10^{6} M_{\odot}[56$ and lies at about $8 \mathrm{kpc}$ from the sun. The angular size of its shadow is thus $\bar{\theta}_{S g r A *}=28 \mu \mathrm{as}$. It is worth noting that some supermassive black holes in other galaxies reach similar orders of magnitude. For example, M87 has a central black hole of $3.2 \times 10^{9} M_{\odot}$ at a distance of $15 \mathrm{Mpc}$ from us [57, resulting in a shadow radius of $11 \mu$ as. However, we must be aware that the luminosity of the sources falls down as $d_{O S}^{-2}$, limiting possible gravitational lensing observations. The black hole in the center of our Galaxy was proposed as the best candidate for black hole lensing by Cunningham and Bardeen 53 . Most of the works in this field have thereafter focussed on this nearby massive black hole.

Sgr $\mathrm{A}^{*}$ has been intensively studied in the radio and $\mathrm{X}$ bands, showing the existence of daily flares coming from the inner accretion regions and probably generated by clumps of matter falling down the horizon. As the Galactic center region is optically thick, the near infrared band offers the best opportunity to study stellar sources around Sgr A*. The latter has only been detected in its flare state in the K-band at 15 magnitudes [58. We refer the interested reader to the textbook by Melia [59] for an extensive review summarizing observations and models of Sgr $\mathrm{A}^{*}$ and its environment.

Weak gravitational lensing by Sgr A* on background stars should be quite common 60, though it has never been identified because of the crowded environment. Unfortunately, spin effects are negligible on the primary and secondary images in the WDL regime. This can be deduced by the size of the primary caustic, which shrinks to zero at large distances. In the case of Sgr A*, the size of the primary caustic is only $1170 \mathrm{~km}$ at $r_{S}=100 R_{S \mathrm{ch}}$. It is therefore necessary to consider either sources close to the black hole or higher order images, which are however very faint and appear very close to the shadow of the black hole within its accretion disk 6, 61. In the following subsections, we will discuss several observational proposals which aim at detecting effects beyond the basic WDL in gravitational lensing by Sgr A* or other black holes. 


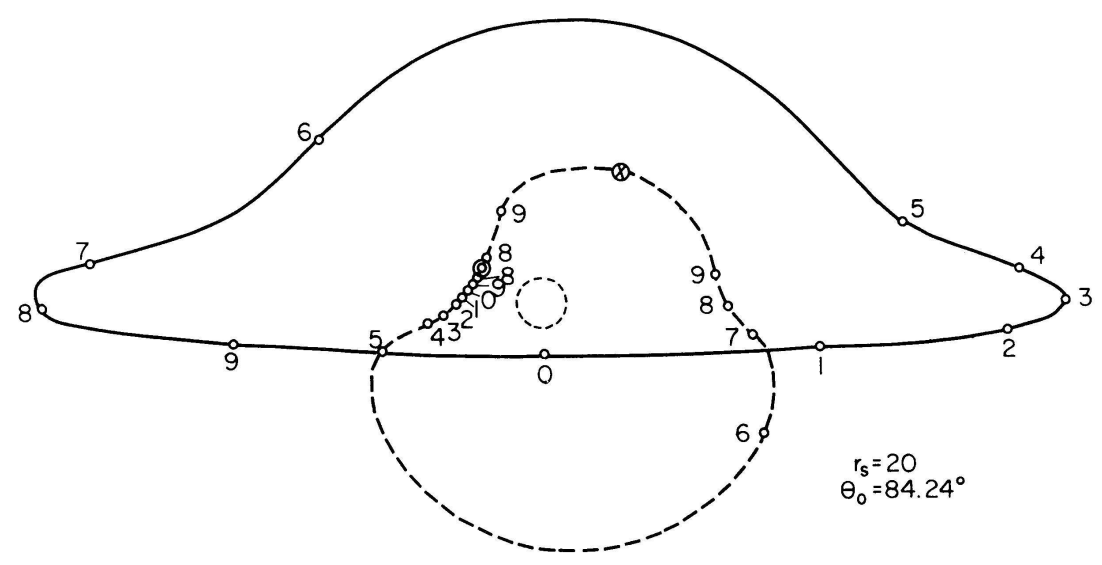

Fig. 9 Apparent positions of the primary (solid line) and secondary (dashed line) images of a source at $r_{S}=20 M$ from an extremal Kerr black hole. The observer is at a polar angle $\vartheta_{O}=84.24^{\circ}$. Ticks mark the positions of the images at 10 equally spaced times. As the source crosses the secondary caustic, two new second order images are formed at the point ( ) and disappear at $\otimes$ when the source exits from the caustic. The inner dashed circle has angular radius $M / r_{O}$. Picture taken from Cunningham and Bardeen (1973) 53.

\subsection{Sources orbiting the black hole}

Stars orbiting the black hole provide natural sources for gravitational lensing. This possibility was first considered by Cunningham and Bardeen [53, who traced the apparent trajectories in the sky of the primary and secondary images for a star orbiting a black hole in a circular orbit. In Fig. 9 we see that the primary image is practically unaffected when the source passes in front of the black hole. When the source transits behind the black hole, the primary image moves around the primary Einstein ring, designing a hat figure. Meanwhile, the secondary image is opposite to the primary image slightly inside the primary Einstein ring. However, when the source moves in front of the black hole, the secondary image is closer to the black hole, approaching the secondary critical curve. Very interestingly, this picture shows the formation of two additional second order images and the annihilation of one of these with the original secondary image. These two events occur when the source enters and exits the giant secondary caustic showed in Fig. 7 r.

We do not know of any stars on a circular equatorial orbit so close to Sgr A*. Yet, the picture of Fig. 9 may be likely applied to a bright spot of hot material lying on the accretion disk. The existence of such features has been proposed by Abramovicz et al. in order to model the rapid X-ray variability of Active Galactic Nuclei (AGN) 62. Substantial amount of work on this possibility has been developed thereafter 38,39,63. Assuming that we do not have enough angular resolution to see the distinct images, the flux of the hot spot would be periodically modulated through gravitational lensing by the black hole. As the spot would rotate very quickly in a strong gravitational 


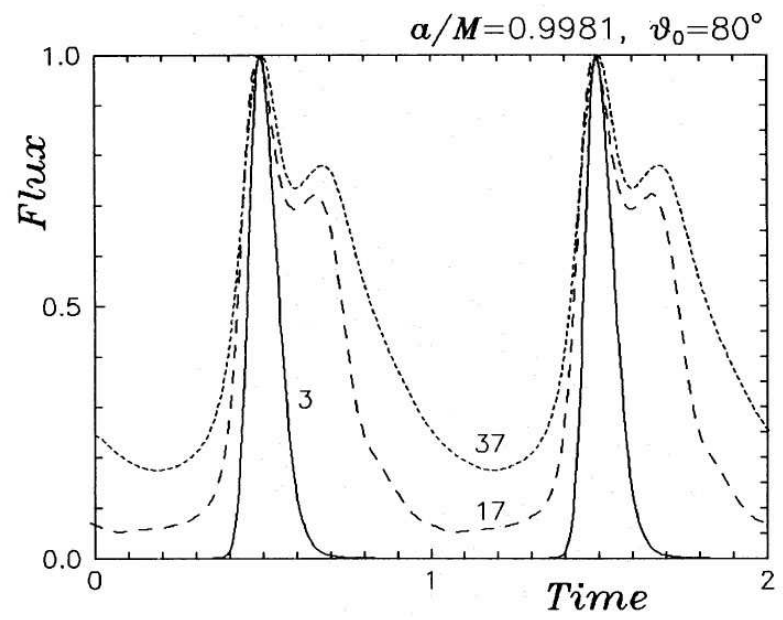

Fig. 10 Light curve of a bright spot orbiting a quasi-extremal Kerr black hole at different radii as indicated in the curve labels. Time is normalized to the period. Picture taken from Karas, Vokrouhlicky and Polnarev (1992) 39.

field environment, Doppler and gravitational red-shift cannot be neglected in a correct treatment. Note that if the disk is opaque to the electromagnetic radiation considered, only the primary image contributes to the observed flux, whereas the second order and all higher order images are stopped by the disk. In Fig. 10, we see that the light curve is dominated by a peak occurring when the spot is close to the primary caustic behind the black hole. This peak may be followed by a secondary peak, depending on the spot distance, caused by Doppler enhancement.

Gravitational lensing of hot spots is very involved because of the large number of parameters to be guessed, such as the size, the shape, the position and the internal velocities of the spot, besides the disk influence on the electromagnetic radiation emitted by the spot. On the other hand, we know of many stars orbiting Sgr A* at larger distances on eccentric orbits [56, 64. Spectra of these stars show that they are early-type unexpectedly young stars; thus, their origin is still under debate. If lensed, these stars would provide much cleaner data 65 . The best candidate is $\mathrm{S} 6$, whose secondary image reaches $K=20.8$, with an angular separation of 0.3 mas from the central black hole 66. This is just beyond the limits of the forthcoming interferometric instrument GRAVITY at VLT [67.

Another interesting possibility comes from Low Mass X-Ray Binaries (LMXB) orbiting Sgr $A^{*} 68$. In these objects, the X-ray radiation is emitted from a very small region, providing good candidates for probing the caustic structure of the black hole in the case it has a non-negligible spin 49. 
4.2 Broad Iron lines in the X-ray spectrum

Among the known manifestations of gravitational lensing by black holes, the broadening of the iron lines is the one that has attracted more interest, as it is accessible with present technology. This phenomenon was first discovered in the Seyfert 1 galaxy MCG-6-30-15 by Tanaka et al. 69, in which a bright emission line is present over the $\mathrm{X}$ continuum at $6.4 \mathrm{keV}$. This line has a characteristic doubly horned structure, with the blue horn higher than the red horn.

This shape has been interpreted as follows: cold material in the accretion disk is ionized by radiation from hot material surrounding it. The following recombination is responsible for the emission line, whose broad structure is due to the strong rotation of the disc, which Doppler shifts the emission. The two horns come from material whose velocity is directed along (or closely to) the line of sight in both ways, with the blue horn appearing more intense thanks to Doppler boosting. This picture also applies to several lines at lower energies, which are generated far from the black hole. The specifity of the iron lines comes from the fact that they probe very high energies. In particular, the $K_{\alpha}$ line at $6.4 \mathrm{keV}$ comes from electrons falling directly to the K-shell. Such energies are only reached in regions very close to the black hole (few Schwarzschild radii). At such distances, general relativistic effects cannot be neglected in the description of the line profiles and the whole spectrum in general [70].

The iron $K_{\alpha}$ line has been observed in several AGNs and the number of works attempting a numerical or analytic description of relativistic iron line profiles has flourished. The first treatments in the Schwarzschild metric have been done in Refs. [71]. Lines in the Kerr metric have been studied in Refs. [72]. Usually, only the primary image of the disk fluorescence is taken into account, because the accretion disk is supposed to be thick. However, the second order image is typically visible if the disc is seen nearly edge-on. The relevance of higher order images has been pointed out in Ref. 73 and later in Ref. 41. A non-exhaustive reference list for later studies is in Ref. 74. Recently, also the L-line at $0.9 \mathrm{keV}$, coming from electrons absorbed to the L-shell, has been observed 75 .

Fig. 11 shows some relativistic line profiles calculated by Fanton et al. [4]. The lines are broader for a disc seen edge-on, showing up to four subpeaks. The line returns single-peaked and is slightly redshifted for a face-on disc. The lines are broader in a Kerr black hole, but there is almost no difference if the inner radius of the emitting region is larger than $6 \mathrm{M}$.

Models of relativistic line profiles depend on many parameters and assumptions on the underlying model, such as opacity, thickness, homogeneity, outer and inner radius of the emitting region, emissivity profile and more and more. It is therefore difficult to use them to extract the black hole parameters with confidence. Nevertheless, they witness the existence of accretion disks at few Schwarzschild radii of distance from the black hole and, at present, they represent the cleaner information we can get from these mysterious regions. 

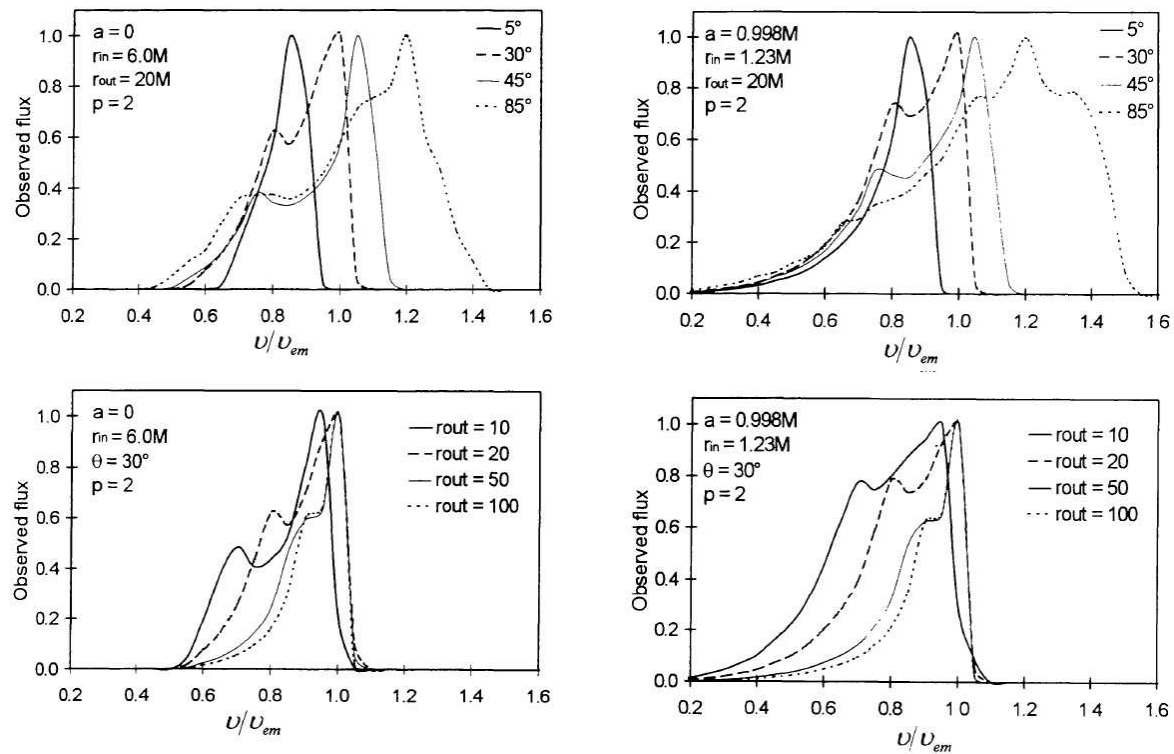

Fig. 11 Relativistic line profiles for emitting disks around a Schwarzschild (left panels) and a quasi-extremal Kerr (right panels) black hole. In the top panels, line profiles for various viewing angles $\vartheta_{O}$ are shown. In the bottom panels, the outer radius of the disk $r_{o u t}$ is varied. Picture taken from Fanton et al. (1997) [41].

\subsection{Direct imaging}

Direct imaging of a black hole with a resolution of the order of the angular size of the Schwarzschild radius is one of the ancient dreams of astrophysicists. Up to now, we have always dealt with indirect information on the regions surrounding the horizon. For example, the measured spectra are the superposition of signals coming from different regions experiencing different physics, from the interstellar environment to the accretion flow up to possible jets. Direct imaging would disentangle these signals and allow a separate study of the different components, unveiling the secrets of the black hole environment.

The first studies on the realistic appearance of a Schwarzschild black hole surrounded by a Keplerian disk date back to Luminet 14, who showed how the disk on the prograde side of the black hole appears brighter thanks to the Doppler boost (which was also responsible of the asymmetry in the iron line profiles, as we have seen in the previous subsection). Fukue and Yokoyama drew pictures at different wavelengths 76 . Viergutz considered a vertically extended disk around a Kerr black hole [40, whereas the first pictures of the realistic appearance of a Kerr black hole were presented by Fanton et al. 41. Afterwards, Falcke, Melia and Agol presented pictures of spherical inflow models taking into account the electron scattering in the $\mathrm{mm}$ band $[77$. Polarimetric images were presented in Ref. [78. A non-exhaustive list of later references is in Ref. 79. 

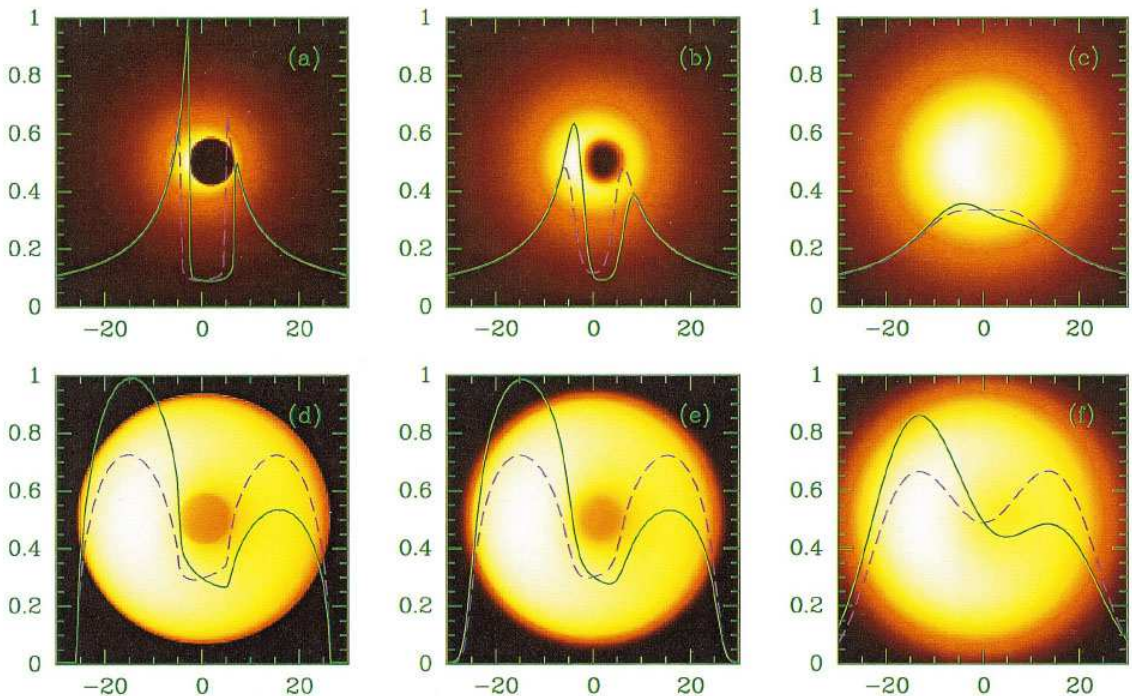

Fig. 12 Top panels: extremal Kerr black hole with radial inflow. Bottom panels: Schwarzschild black hole with flow on Keplerian shells. Left panels: unscattered plots. Middle panels: the same plots including scattering by interstellar medium at $\lambda=0.6 \mathrm{~mm}$. Right panels: the same plots at $\lambda=1.3 \mathrm{~mm}$. Picture taken from Falcke, Melia and Agol (1999) 77 .

In Fig. 12 we report the pictures by Falcke, Melia and Agol 77]. The difference between Kerr and Schwarzschild is mostly in the shape of the shadow, which is flattened on the prograde side for Kerr. The prograde side of the flow is Doppler-boosted in both cases, giving poor indication on the spin of the black hole itself. We can also appreciate the blurring caused by scattering on electrons in the interstellar medium. Very Long Baseline Interferometry (VLBI) observations in the $\mathrm{cm}$ band show that the structure of Sgr $\mathrm{A}^{*}$ is washed out by scattering, with its apparent size scaling with the wavelength as $\lambda^{2}$ [80. Its intrinsic structure should be accessible at sub-mm wavelengths, on which Ref. 77 focussed.

The best angular resolution so-far achieved by human instrumentations has been reached at $\lambda=3 \mathrm{~mm}$ by VLBI on an intercontinental scale, with 18 $\mu$ as 81 . Even if this is less than the apparent shadow of Sgr A*, interstellar scattering is still too high at this wavelength. Observations of M87 at this wavelength have also been carried out 82 . This has inspired the idea that the black hole in M87 could be an even more interesting target than Sgr A* 83], thanks to its jet structure. Meanwhile, projects for pushing the global network of VLBI to shorter wavelengths are under discussion 84 and represent the most promising frontier for direct imaging of black holes. Indeed, present observations at $\lambda=1.3 \mathrm{~mm}$ seem to point that the intrinsic size of the emitting region of $\operatorname{Sgr} \mathrm{A}^{*}$ is smaller than the shadow size [85. This is compatible with 
a black hole surrounded by an accretion disk whose prograde side luminosity is enhanced by Doppler boost (see Fig. 12) 86].

Dynamical features such as hot spots rotating around the black hole may be directly imaged in the future 87]. These could also be detected at worst resolutions by following the motion of the centroid of Sgr $A^{*}$ [88.

In the near infrared bands, the best resolution is now obtained by the Keck interferometer and the VLTI. The latter will be upgraded with the new instrument GRAVITY 67, which will take the resolution to 3 mas in the Kband. This is far from the shadow scale of the black hole but is a great advance for the determination of stellar orbits, which put the tighter constraint to the mass of Sgr A*. It is also important for the study of flares in the infrared.

Finally, the MAXIM project for developing X-ray interferometry on spacecraft has been under study for several year:20. The incredible difficulties posed by this challenge will be rewarded by a black hole imager with resolution even better than $1 \mu$ as. The new frontiers opened by such a futuristic instrument can hardly be imagined.

Acknowledgements I wish to thank Mauro Sereno, Luigi Mancini, Arlie Petters and Eric Agol for useful suggestions and comments on the manuscript. I acknowledge support by Agenzia Spaziale Italiana, by PRIN (Prot. 2008NR3EBK_002), and by research funds of Salerno University.

\section{References}

1. C. Darwin, Proc. of the Royal Soc. of London A, 249, 180 (1959).

2. S. Weinberg, "Gravitation and Cosmology", John Wiley \& Sons, New York (1972).

3. C.-M. Claudel, K.S. Virbhadra and G.F.R. Ellis, Jour. of Math. Phys., 42, 818 (2001).

4. R. Atkinson, Astron. J, 70, 517 (1965).

5. H.C. Ohanian, Am. J. Phys., 55, 428 (1987).

6. K.S. Virbhadra and G.F.R. Ellis, Phys. Rev. D, 62, 084003 (2000).

7. V. Bozza, S. Capozziello, G. Iovane, and G. Scarpetta, Gen. Rel. and Grav., 33, 1535 (2001).

8. S. Frittelli and E.T. Newman, Phys. Rev. D, 59, 124001 (1999).

9. S. Frittelli, T.P. Kling, and E.T. Newman, Phys. Rev. D, 61, 064021 (2000).

10. V. Perlick, Phys. Rev. D, 69, 064017 (2004).

11. V. Perlick, Living Rev. Relativity, 7, 9 (2004).

12. R. Nemiroff, Am. Jour. Phys., 61, 619 (1993).

13. V. Bozza, Phys. Rev. D, 78, 103005 (2008).

14. J.P. Luminet, A\&A, 75, 228 (1979).

15. D.E. Holz and J.A. Wheeler, ApJ, 578, 330 (2002).

16. Y. Décanini and A. Folacci, Phys. Rev. D, 81, 024031 (2010).

17. R. Epstein and I. Shapiro, Phys. Rev. D, 22, 2947 (1980);

18. E. Fischbach and B.S. Freeman, Phys. Rev. D, 22, 2950 (1980); G. Richter and R. Matzner, Phys. Rev. D, 26, 1219 (1982); 26, 2549 (1982); A.F. Sarmiento, Gen. Rel. and Grav., 14, 793 (1982); M. Sereno, Phys. Rev. D, 67, 064007 (2003).

19. C. R. Keeton and A. O. Petters, Phys. Rev. D 72, 104006 (2005); 73, 044024 (2006); Phys. Rev. D 73, 104032 (2006).

20. J. Ebina, T. Osuga, H. Asada, and M. Kasai, Prog. of Th. Phys., 104, 1317 (2000); G.F. Lewis, X.R. Wang, Prog. of Th. Phys., 105, 893 (2001).

20 http://maxim.gsfc.nasa.gov. 
21. M. Sereno, Phys. Rev. D, 69, 023002 (2004)

22. Th. Damour and G. Esposito-Farèse, Phys. Rev. D, 53, 5541 (1996).

23. M. Sereno, Phys. Rev. D, 77, 043004 (2008).

24. S. Chandrasekhar, "Mathematical Theory of Black Holes", Clarendon Press, Oxford (1983).

25. E.F. Eiroa, G.E. Romero and D.F. Torres, Phys. Rev. D, 66, 024010 (2002).

26. V. Bozza, Phys. Rev. D, 66, 103001 (2002).

27. E.F. Eiroa and D.F. Torres, Phys. Rev. D, 69, 063004 (2004).

28. V. Bozza and G. Scarpetta, Phys. Rev. D, 76, 083008 (2007).

29. A. Bhadra, Phys. Rev. D 67, 103009 (2003); E. F. Eiroa, Phys. Rev. D 71, 083010 (2005); R. Whisker, Phys. Rev. D 71, 064004 (2005); A. S. Majumdar and N. Mukherjee, Int. J. Mod. Phys. D 14, 1095 (2005); Eiroa, Phys. Rev. D, 73, 043002 (2006); K. K. Nandi, Y.-Z. Zhang, and A. V. Zakharov, Phys. Rev. D, 74, 024020 (2006); K. Sarkar and A. Bhadra, Class. Quant. Grav. 23, 6101 (2006); N. Mukherjee, A.S. Majumdar, Gen. Rel. and Grav., 39, 583 (2007); F. Rahaman, M. Kalam and S. Chakraborty, Chin. J. Phys., 45, 518 (2007); T. K. Dey, S. Sen, Mod. Phys. Lett. A, 23, 953 (2008); S. Chen, J. Jing, Phys. Rev. D, 80, 024036 (2009); T. Ghosh and S. Sengupta, Phys. Rev. D, 81, 044013 (2010).

30. V. Bozza, Nuovo Cimento B, 122, 547 (2007).

31. S.V. Iyer and A.O. Petters, Gen. Rel. and Grav., 39, 1563 (2007).

32. V. Bozza and L. Mancini, Gen. Rel. and Grav., 36, 435 (2004).

33. K. Chang and S. Refsdal, Nat., 282, 561 (1979); A\& A, 132, 168 (1984).

34. E. Poisson, Phys. Rev. Lett., 94, 161103 (2005).

35. V. Bozza and M. Sereno, Phys. Rev. D, 73, 103004 (2006).

36. P. Amore and S. Arceo, Phys. Rev. D, 73, 083004 (2006); P. Amore, S. Arceo, and F.M. Fernandez, Phys. Rev. D, 74, 083004 (2006); P. Amore, M. Cervantes, A. De Pace, and F.M. Fernandez, Phys. Rev. D, 75,083005 (2007).

37. B. Carter, Phys. Rev., 174, 1559 (1968).

38. K.P. Rauch and R.D. Blandford, ApJ, 421, 46 (1994).

39. V. Karas, D. Vokrouhlicky and A.G. Polnarev, MNRAS, 259, 569 (1992).

40. S.U. Viergutz, A\&A, 272, 355 (1993).

41. C. Fanton, M. Calvani, F. de Felice, and A. Cadez, PASJ, 49, 159 (1997).

42. J. Dexter and E. Agol, ApJ, 696, 1616 (2009).

43. S.V. Iyer and E.C. Hansen, Phys. Rev. D, 80, 124023 (2009).

44. S. Pineault and R.C. Roeder, ApJ, 212, 541 (1977); 213, 548 (1977).

45. I. Bray, Phys. Rev. D, 34, 367 (1986); A. Edery and J. Godin, Gen. Rel. and Grav., 38, 1715 (2006); M.C. Werner and A. O. Petters, Phys. Rev. D, 76, 064024 (2007)

46. H. Asada and M. Kasai, Prog. Theor. Phys., 104, 95 (2000); H. Asada, M. Kasai, and T. Yamamoto, Phys. Rev. D, 67, 043006 (2003).

47. M. Sereno and F. De Luca, Phys. Rev. D, 74, 123009 (2006); 78, 023008 (2008).

48. S.E. Vazquez and E.P. Esteban, Nuovo Cim. B, 119, 489 (2004); V. Bozza, Phys. Rev. D, 67, 103006 (2003).

49. V. Bozza, F. De Luca, G. Scarpetta, and M.Sereno, Phys. Rev. D 72, 083003 (2005); V. Bozza, F. De Luca, and G. Scarpetta, Phys. Rev. D 74, 063001 (2006).

50. A. de Vries, Class. Quant. Grav., 17, 123 (2000); C. Bambi and K. Freese, Phys. Rev. D 79, 043002 (2009).

51. J. Bardeen, in C. De Witt and B.S. De Witt, "Black Holes. École d'été de Physique Théorique, Les Houches 1972". Gordon \& Breach Science Publishers, New York (1973);

52. R. Takahashi, ApJ, 611, 996 (2004); Publ. Astron. Soc. Japan, 57, 273, (2005); A.F. Zakharov et al., A\& A, 442, 795 (2005); K. Hioki and K.-I. Maeda, Phys. Rev. D, 80, 024042 (2009).

53. C.T. Cunningham and J.M. Bardeen, ApJ 173, L137 (1972); ApJ 183, 237 (1973).

54. V. Bozza, Phys. Rev. D, 78, 063014 (2008).

55. D. Richstone et al., Nature, 395, A14 (1998).

56. S. Gillessen et al., ApJ, 692, 1075 (2009).

57. F. Macchetto et al., ApJ, 489, 579 (1997).

58. R. Genzel al., Nature, 425, 934 (2003). 
59. F. Melia, "The Black Hole at the center of our Galaxy", Princeton University Press, (2003).

60. M. Wardle and F. Yusuf-Zadeh, ApJ, 387, L65 (1992); M. Jaroszyński, Acta Astron., 48, 413, (1998); T. Alexander and A. Sternberg, ApJ, 520, 137 (1999); T. Alexander, ApJ, 553, L149 (2001); J. Chanamé, A. Gould, and J. Miralda-Escudé, ApJ, 563, 793 (2001); T. Alexander and A. Loeb, ApJ, 551, 223 (2001); A. Nusser and T. Broadhurst, MNRAS, 355, L6, (2004).

61. A.O. Petters, MNRAS, 338, 457 (2003).

62. M.A. Abramovicz, A. Lanza, E.A. Spiegel, and E. Szuszkiewicz, Nature, 356, 41 (1992)

63. V. Karas and G. Bao, A\&A, 257, 531 (1992); G. Bao, A\&A, 257, 594 (1992); A.F.

Zakharov, MNRAS, 269, 283 (1994); G. Bao, P. Hadrava, and E. Østgaard, ApJ, 425, 63 (1994); G. Bao and E. Østgaard, ApJ, 443, 54 (1995); Hollywood et al., ApJ, 448, L21 (1995); V. Karas, ApJ, 470, 743 (1996); MNRAS, 288, 12 (1997); PASJ, 51, 317 (1999); J. Fukue, PASJ, 55, 1121 (2003).

64. R. Schödel et al., ApJ, 596, 1015 (2003); A.M. Ghez et al., ApJ, 620, 744 (2005).

65. F. De Paolis, A. Geralico, G. Ingrosso, and A.A. Nucita, A\&A, 409, 809 (2003); V. Bozza and L. Mancini, ApJ, 611, 1045 (2004); 627, 790 (2005).

66. V. Bozza and L. Mancini, ApJ, 696, 701 (2009).

67. Eisenhauer et al., Proceedings of the SPIE, 7013, 70132 (2008).

68. M.P. Muno et al., ApJ 622, L113 (2005).

69. Y. Tanaka et al., Nature, 375, 659 (1995).

70. C.T. Cunningham, ApJ, 202, 788 (1975); I. Asaoka, PASJ, 41, 763 (1989); M. Jaroszyński and A. Kurpiewski, A\&A, 326, 419 (1997).

71. A.C. Fabian, M.J. Rees, L. Stella, and N.E. White, MNRAS, 238, 729 (1989); L. Stella, Nature, 344, 747 (1990); A. Laor, ApJ, 376, 90 (1991).

72. Y. Kojima, MNRAS, 250, 629 (1991); K. Chen and D. Eardley, ApJ, 382, 125 (1991); G. Bao, ApJ, 409, L41 (1993), G. Matt, G.C. Perola, and L. Stella, A\&A, 267, 643 (1993); J.-M. Hameury, J.-A. Marck, and D. Pelat, A\&A, 287, 795 (1994).

73. G. Bao, P. Hadrava, and E. Østgaard, ApJ, 435, 55 (1994).

74. G. Bao, P. Hadrava, and E. Østgaard, ApJ, 464, 684; B.C. Bromley; K. Chen, and W.A. Miller, ApJ; 475, 57 (1997); Ch.S. Reynolds, A.J. Young, M.C. Begelman, and A.C. Fabian, ApJ, 514, 164 (1999); A. Martocchia, V. Karas, and G. Matt, MNRAS, 312, 817 (2000); Q. Yu, Y. Lu, MNRAS, 311, 161 (2000); M. Dovciak, V. Karas, and T. Yaqoob, ApJS, 153, 205 (2004); K. Beckwith and Ch. Done, MNRAS, 352, 353 (2004); K. Fukumura and S. Tsuruta, ApJ, 613, 700 (2004); B. Czerny et al., A\&A, 420, 1 (2004); A. Cadež and M. Calvani, MNRAS, 363, 177 (2005); R.W. Goosmann, A\&A, 454, 741 (2006); A.F. Zakharov and S.V. Repin, New Astr., 11, 405 (2006); S.V. Fuerst and K. Wu, A\&A, 474, 55 (2007); S.V. Repin, V.N. Lukash, and V.N. Strokov, Astr. Reps., 52, 1 (2008).

75. A.C. Fabian et al., Nature, 459, 540 (2009)

76. J. Fukue and T. Yokoyama, PASJ, 40, 15 (1988).

77. H. Falcke, F. Melia, and E. Agol, ApJ, 528, L13 (1999).

78. B.C. Bromley, F. Melia, and S. Liu, ApJ, 555, L83 (2001);

79. J. Fukue, PASJ, 55, 155 (2003); Takahashi, ApJ, 611, 996 (2004); K. Beckwith and C. Done, MNRAS, 359, 1217 (2005); A.E. Broderick and R. Narayan, ApJ, 638, L21 (2006); F. Yuan, Zh.-Q. Shen, and L. Huang, ApJ, 642, L45 (2006); L. Huang, M. Cai, Zh.-Q. Shen, and F. Yuan, MNRAS, 379, 833 (2007); Y.-F. Yuan, Z. Cao, L. Huang, and Zh.-Q. Shen, ApJ, 699, 722 (2009).

80. K.Y. Lo et al., ApJ, 508, L61 (1998).

81. T.P. Krichbaum et al., Proc. 6th European VLBI Network Symposium, Bonn, Germany (2002).

82. T.P. Krichbaum et al., Jour. of Phys. Conf. Ser., 54, 328 (2006).

83. A.E. Broderick and A. Loeb, ApJ, 697, 1164 (2009).

84. T.P. Krichbaum et al., arXiv:0812.4211

85. Sh.S. Doeleman et al., Nature, 455, 78 (2008).

86. J. Dexter, E. Agol, and P. Ch. Fragile, APJ, 703, L142 (2009).

87. A.E. Broderick and A. Loeb, MNRAS, 363, 353 (2005); 367, 905 (2006).

88. A.E. Broderick and A. Loeb, ApJ, 636, L109 (2006). 